\title{
Minimal scenario of criticality for electroweak scale, neutrino masses, dark matter, and inflation
}

\author{
Yuta Hamada $^{1, \mathrm{a}}$, Hikaru Kawai ${ }^{2, \mathrm{~b}}$, Kiyoharu Kawana ${ }^{3, \mathrm{c}}$, Kin-ya Oda ${ }^{4, \mathrm{~d}}$, Kei Yagyu ${ }^{4, \mathrm{e}}$ \\ ${ }^{1}$ Department of Physics, Harvard University, Cambridge, MA 02138, USA \\ 2 Department of Physics and Center for Theoretical Physics, National Taiwan University, Taipei 106, Taiwan, ROC \\ ${ }^{3}$ Department of Physics and Astronomy, Center for Theoretical Physics, Seoul National University, Seoul 08826, Korea \\ ${ }^{4}$ Department of Physics, Osaka University, Osaka 560-0043, Japan
}

Received: 30 May 2021 / Accepted: 10 October 2021 / Published online: 2 November 2021

(C) The Author(s) 2021

\begin{abstract}
We propose a minimal model that can explain the electroweak scale, neutrino masses, Dark Matter (DM), and successful inflation all at once based on the multicriticalpoint principle (MPP). The model has two singlet scalar fields that realize an analogue of the Coleman-Weinberg mechanism, in addition to the Standard Model with heavy Majorana right-handed neutrinos. By assuming a $Z_{2}$ symmetry, one of the scalars becomes a DM candidate whose property is almost the same as the minimal Higgs-portal scalar DM. In this model, the MPP can naturally realize a saddle point in the Higgs potential at high energy scales. By the renormalization-group analysis, we study the critical Higgs inflation with non-minimal coupling $\xi|H|^{2} R$ that utilizes the saddle point of the Higgs potential. We find that it is possible to realize successful inflation even for $\xi=25$ and that the heaviest right-handed neutrino is predicted to have a mass around $10^{14} \mathrm{GeV}$ to meet the current cosmological observations. Such a small value of $\xi$ can be realized by the Higgs-portal coupling $\lambda_{S H} \simeq 0.32$ and the vacuum expectation value of the additional neutral scalar $\langle\phi\rangle \simeq 2.7 \mathrm{TeV}$, which correspond to the dark matter mass $2.0 \mathrm{TeV}$, its spinindependent cross section $1.8 \times 10^{-9} \mathrm{pb}$, and the mass of additional neutral scalar $190 \mathrm{GeV}$.
\end{abstract}

\section{Introduction}

The observed Higgs mass supports the assumption that the Standard Model (SM) is not much altered up to the Planck

\footnotetext{
a e-mail: yhamada@fas.harvard.edu

be-mail: hkawai@gauge.scphys.kyoto-u.ac.jp

c e-mail: kawana@snu.ac.kr (corresponding author)

de-mail: odakin@phys.sci.osaka-u.ac.jp

e e-mail: yagyu@het.phys.sci.osaka-u.ac.jp
}

scale. More precisely, the critical value of the top-quark pole mass is about $m_{t \text {,critical }}^{\text {pole }} \simeq 171.4 \mathrm{GeV}$ [1] for the theoretical border between stability and instability (or metastability) of the effective Higgs potential for the observed Higgs mass $\simeq 125 \mathrm{GeV}$; see also Refs. [2-4]. ${ }^{1}$ This critical value of the top pole mass is consistent at the $1.4 \sigma$ level with the latest combination of the experimental results $m_{t}^{\text {pole }}=172.4 \pm 0.7$ $\mathrm{GeV}$ [5]. Surprisingly, the degenerate minimum of the Higgs potential at the critical top mass coincides with the Planck scale, and such a behavior of the potential has a lot of implications to high energy physics and cosmology. This interesting behavior of the Higgs potential can be understood by the multicritical-point principle (MPP) [6-16] (see also Refs. $[17,18])$ that "coupling constants that are relevant at low energies are tuned to a multicritical point around which the vacuum structure drastically changes when they are varied." We note that existence of a saddle-point around the Planck scale, rather than a degenerate vacua, is another possible form of multicriticality. This fact is used in the critical Higgs inflation [1,19-22] explained below.

Besides such interesting behavior of the Higgs potential, there are many mysteries and problems in particle physics and cosmology. For example, we have not yet understood the origin of electroweak (EW) scale $v=246 \mathrm{GeV}$, which is hugely small compared to the Planck or string scale $10^{18} \mathrm{GeV}$ at which people believe that there must exist an unified theory which includes quantum gravity. Further, the Majorana-mass scale for the right-handed neutrinos is unknown in the SM with the seesaw mechanism [23-27]. Moreover, the recent observations in cosmology, including that of the cosmic microwave background (CMB), have established the existence of (cold) dark matter (DM). It motivates us to consider

\footnotetext{
1 With the current central value $m_{H}=125.1 \pm 0.1 \mathrm{GeV}$ [5], the critical top mass becomes $m_{t \text {, critical }}^{\text {pole }} \simeq 171.2 \mathrm{GeV}$ [4].
} 
a new particle whose interactions with the SM particles are relatively weak.

In addition, the CMB fluctuations may also provide hints for further new physics since they are seeded at high energy scales during inflation. Current observation is consistent with single-field inflation models, among which the Higgs inflation provides one of the best fits [28-31]. In particular, the critical Higgs inflation is inspired by the possible existence of the saddle point around the Planck scale in the MPP, which helps to flatten the Higgs potential and allows rather small value of the non-minimal coupling $\xi=\mathcal{O}(10)$ in $\xi|H|^{2} R$. (The not-so-large coupling is favorable from unitarity [3236].)

In this paper, we consider the most economical model that can simultaneously explain all the above issues: the critical Higgs inflation, neutrino masses, EW scale, and DM. ${ }^{2}$ The model consists of two additional real singlet scalar fields and the SM with the right-handed neutrinos. As a result, we manage to predict the parameters in a well determined narrow region by taking into account the constraints from the DM relic abundance, its direct detection experiments, the CMB fluctuations, and the latest LHC data, while keeping the perturbativity up to the Planck scale. We find that the Higgs-portal coupling and the vacuum expectation value of the additional neutral scalar are fixed to be $\lambda_{S H} \simeq 0.32$ and $\langle\phi\rangle \simeq 2.7 \mathrm{TeV}$, resulting in the dark matter mass $2.0 \mathrm{TeV}$, its spin-independent cross section $1.8 \times 10^{-9} \mathrm{pb}$, and the mass of additional neutral scalar $190 \mathrm{GeV}$.

The motive behind this model is as follows. If $m_{t}^{\text {pole }}>$ $m_{t, \text { critical }}^{\text {pole }}$, the instability of the Higgs potential requires additional positive contributions to the renormalization group (RG) of the Higgs quartic coupling $\lambda_{H}$. The simplest possibility is to introduce a real scalar field $S$ that couples to the SM Higgs doublet $H$ via a quartic interaction $\lambda_{S H}|H|^{2} S^{2}$. (This is nothing but the Higgs-portal DM model [38-41].) However, such an extension could yield too large a tensorto-scalar ratio of the $\mathrm{CMB}$ in the (critical) Higgs inflation, because of the raised Higgs potential at high scales; see e.g. $[22,42]$. We can resolve this issue by introducing additional superheavy fermions that lowers the Higgs potential at higher scales, while keeping the above mentioned positive contribution from the scalar at lower scales. Therefore, it is reasonable to assume a high-scale seesaw in which right-handed neutrinos have large Majorana masses $M_{R}$ and large Yukawa couplings $y_{v}$. In fact, it is possible to maintain the saddle point of the Higgs potential at high energy scales in the existence of new scalar field(s) when $M_{R} \sim 10^{14} \mathrm{GeV}$ [22]. This is also one of the interesting predictions of the MPP.

\footnotetext{
2 Quite recently, a similar scenario to simultaneously explain these problems has been studied in Ref. [37] in the context of the classical scale invariance, in which a fermionic DM candidate is considered. In our scenario, we consider the MPP and a scalar DM candidate.
}

So far, the MPP has not explained the origin of the EW scale. In fact, we may do so as follows. It is known that the Coleman-Weinberg (CW) mechanism [43] can naturally explain the hierarchy between the EW and Planck scales through the dimensional transmutation. Important assumption behind the $\mathrm{CW}$ mechanism is that the renormalized mass-squared parameter vanishes at the origin of the scalar field space. This assumption is called the classical scale invariance (CSI) [44-56]. ${ }^{3}$ On the other hand, such CSI-type potentials can be naturally understood as one of the possible multicriticities in MPP without referring to scale invariance $[61,62] .{ }^{4}$ From this MPP point of view, the simplest realization of the dimensional transmutation is achieved by only two real singlet scalar fields, one of which is $S$ mentioned above [61,62].

Although we will focus on a specific model proposed in $[61,62]$ (namely only the CP 2-2 among various multicritical points in the parameter space) in the following, the analysis of Higgs inflation does not much depend on the details of the model because only the scalar coupling $\lambda_{S H}$ and the neutrino Yukawa $y_{v}$ play important roles to determine the behaviours of the Higgs potential at high energy scales. In this sense, the same analysis is easily applicable to similar extensions of the SM.

The organization of the paper is as follows. In Sect. 2, we briefly explain the minimal model of dimensional transmutation $[61,62]$ extended with right-handed neutrinos and study the RG. In Sect. 3, we study the saddle point of the Higgs potential at high scales. In Sect. 4, we discuss the critical Higgs inflation. In Sect. 5, we show the method and results for our numerical prediction for the inflationary observables. Summary and discussion are given in Sect. 6. In Appendix $\mathrm{A}$, we list the two-loop renormalization group equations (RGEs). In Appendix B, we summarize basic results for a general single-field slow-roll inflation. In Appendix $\mathrm{C}$, we summarize basic results for the ordinary (non-critical) Higgs inflation. In Appendix D, we show analytic results of expansion around the saddle point.

\section{Model}

In this section, we introduce the minimal model for the EW scale, neutrino masses, DM and the critical Higgs inflation. The model is based on $[61,62]$ whose scalar sector contains two additional real singlet scalars $S$ and $\phi$. We also take into account heavy Majorana right-handed neutrinos $v_{R}^{i}$ with

\footnotetext{
3 In Refs. [57,58], the bare Higgs mass too is required to vanish; see also Ref. $[59,60]$ for the discussion on bare mass in the SM.

4 Throughout this paper, we call a potential that has vanishing first and second derivatives at the origin in the field space, $\left.V^{\prime}\right|_{\phi=0}=\left.V^{\prime \prime}\right|_{\phi=0}=$ 0 , the CSI-type potential.
} 

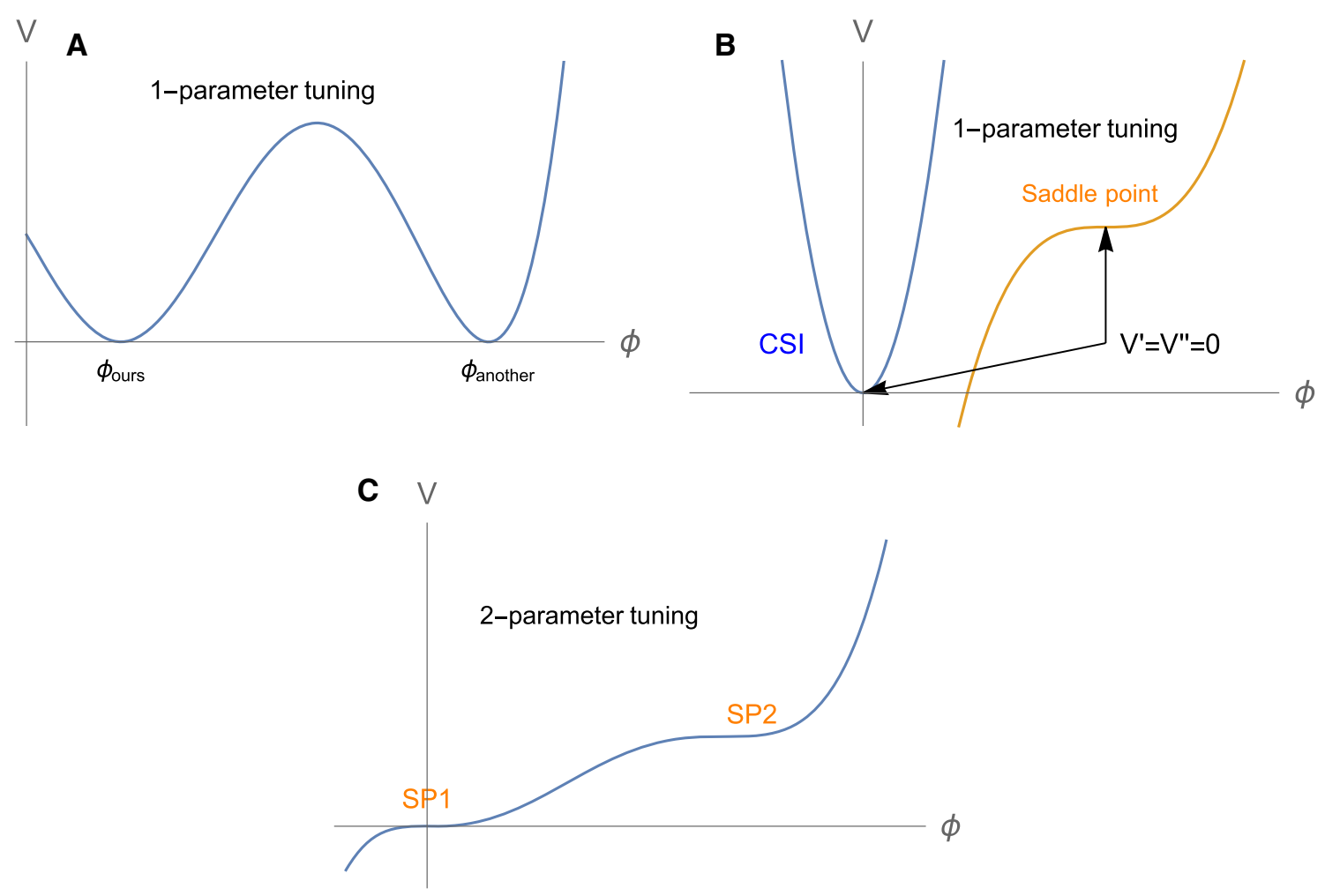

Fig. 1 Typical examples of the effective potential resulting from the MPP

three generations. In order to make $S$ a DM candidate, we impose the $Z_{2}$ symmetry $S \rightarrow-S$, with all the other fields being invariant.

Here we briefly review the consequence of the MPP, focusing on what kind of effective potentials the MPP can result in. Before proceeding, we first note that the effective potential exists independently of the renormalization scale, up to the renormalization of the field, for a given set of bare parameters. (In other words, the renormalization scale dependence should only arise due to truncation at some loop level.) In Fig. 1, we list examples of the possible effective potentials resulting from the MPP: In panel A, we show the first kind of tuning $V_{\phi=\phi_{\text {ours }}}=V_{\phi=\phi_{\text {another }}}$ given in the original version of the MPP [6-8]. In panel B, we show another one-parameter tuning $V^{\prime}=V^{\prime \prime}=0$, which provides an explanation for the CSI-type potential $\left.V^{\prime}\right|_{\phi=0}=\left.V^{\prime \prime}\right|_{\phi=0}=0$. This is also the key assumption for the Coleman-Weinberg mechanism. From this point of view, the CSI-type potential is just one of the possible critical points, in the theory space, in the MPP. In panel C, we show the potential (called CP 2-2) that is a consequence of two-parameter tuning to realize two saddle points $\left.V^{\prime}\right|_{\mathrm{SP} 1}=\left.V^{\prime \prime}\right|_{\mathrm{SP} 1}=0$ and $\left.V^{\prime}\right|_{\mathrm{SP} 2}=\left.V^{\prime \prime}\right|_{\mathrm{SP} 2}=0$. We stress that all the above potentials are achieved in the context of the MPP irrespectively of any kind of scale invariance. Therefore, it does not matter whether other fields such as the right-handed neutrinos have mass parameters in the action or not.
Among the various critical points analyzed so far, we take the CP 2-2 defined in Ref. [62] that has two saddle points in the effective potential, because the widest parameter region is allowed by the constraints considered. The renormalized Lagrangian is

$$
\begin{aligned}
\mathcal{L}= & \mathcal{L}_{\mathrm{SM}}-\frac{1}{2}\left(\partial_{\mu} \phi\right)^{2}-\frac{1}{2}\left(\partial_{\mu} S\right)^{2} \\
& -\lambda_{H}\left(H^{\dagger} H\right)^{2}-\frac{\lambda_{\phi}}{4 !} \phi^{4}-\frac{\lambda_{\phi S}}{4} \phi^{2} S^{2}-\frac{\lambda_{S}}{4 !} S^{4} \\
& +\frac{\lambda_{\phi H}}{2} \phi^{2}\left(H^{\dagger} H\right)-\frac{\lambda_{S H}}{2} S^{2}\left(H^{\dagger} H\right)-\frac{\mu_{\phi}}{3 !} \phi^{3} \\
& +\frac{1}{2} \sum_{i=1}^{3} \overline{v_{R i}} \gamma^{\mu} i \partial_{\mu} v_{R i}-\frac{1}{2} \sum_{i=1}^{3} M_{R i} \overline{\nu_{R i}{ }^{c}} \nu_{R i} \\
& -\sum_{i, j=1}^{3}\left(y_{v i j} \overline{L_{i}} H^{c} v_{R j}+\frac{y_{i j}^{\phi}}{2} \phi \overline{v_{R i}{ }^{c}} v_{R j}+\text { h.c }\right),
\end{aligned}
$$

where $\mathcal{L}_{\mathrm{SM}}$ is the SM Lagrangian without the Higgs potential and without the right-handed neutrinos, and $H^{c} \equiv i \sigma_{2} H^{*}$. Note that all the couplings in the Lagrangian are defined by the zero-momentum subtraction scheme. These couplings are the same as those in the effective potential. It is precisely these couplings that are restricted by the MPP. Here, among several realizations of the MPP, we choose a class of simple solutions that satisfy the vanishing of all the terms with dimensionful 
couplings except for $\phi^{3}$, namely, $\phi, \phi^{2}, S^{2}, H^{\dagger} H, \phi H^{\dagger} H$, and $\phi S^{2}$. In Eq. (1), we have omitted these terms from the beginning. Note that the renormalized mass of $S$ around the origin $h=\phi=S=0$ is set to zero by the MPP, while it obtains the finite mass around the true vacuum as we will see in Eq. (9).

For simplicity, we will make the following three assumptions. First, we take $\lambda_{S}$ to be zero at the EW scale since it is irrelevant for our discussion. ${ }^{5}$ Second, we assume that the Majorana Yukawa couplings $y_{i j}^{\phi}$ are not large and do not affect the other RG runnings. ${ }^{6}$ Third, we assume that Majorana masses are all degenerate each other, $M_{R 1}=M_{R 2}=$ $M_{R 3}=M_{R}$, and neglect the mixing in the neutrino Dirac Yukawa couplings, i.e., $y_{v i j}=y_{v i} \delta_{i j}$. At the leading order of $M_{R}^{-1}$, the neutrino mass matrix becomes

$\left(m_{v}\right)_{i j}=\frac{v^{2}}{2 M_{R}} \delta_{i j} y_{v i}^{2}$

We call the number of "heavier active neutrinos" $n_{v}$, namely the degeneracy of the largest $y_{v i}$ : in the case of normal and inverted hierarchies, we have $n_{v}=1$ and 2, respectively, and when all the neutrino masses are degenerate, we have $n_{v}=3$. Throughout this paper, we will use $m_{v}=0.05 \mathrm{eV}$ [5] as the heaviest mass of active neutrinos. ${ }^{7}$ The relation between $M_{R}$ and the largest $y_{v}$ is then determined to be $y_{v} \simeq$ $0.41 \sqrt{M_{R} / 10^{14} \mathrm{GeV}}$.

Here, we briefly review how the $\mathrm{CW}$ mechanism realizes the EW symmetry breaking in our model with the MPP. The effective potential for the $\phi-H$ system can be expressed at one-loop level as

$$
\begin{aligned}
V_{\text {eff }}= & \lambda_{H}\left(H^{\dagger} H\right)^{2}+\frac{\lambda_{\phi H}}{2} \phi^{2}\left(H^{\dagger} H\right)+\frac{\mu_{\phi}}{3 !} \phi^{3}+\frac{\lambda_{\phi}}{4 !} \phi^{4} \\
& +\frac{M_{\phi}^{4}(\phi)}{64 \pi^{2}}\left[\ln \frac{M_{\phi}^{2}(\phi)}{\mu^{2}}-\frac{1}{2}\right]+\frac{\lambda_{\phi S}^{2} \phi^{4}}{256 \pi^{2}} \\
& \times\left[\ln \frac{\lambda_{\phi S} \phi^{2}}{2 \mu^{2}}-\frac{1}{2}\right]+\Delta V_{1-\text { loop }}(h, \mu),
\end{aligned}
$$

where $M_{\phi}^{2}(\phi)=\mu_{\phi} \phi+\lambda_{\phi} \phi^{2}$, and the renormalization scheme applied in [61] is used. The $\Delta V_{1-\text { loop }}$ term is the 1-loop potential for $h$ given in Eq. (13). In this expression,

\footnotetext{
5 This choice makes the perturbativity bound loosest, while keeping the stability of the effective potential.

6 If one wants, one can forbid it by a $Z_{2}^{\prime}$ symmetry $\phi \rightarrow-\phi$ that is softly broken by the $\phi^{3}$ term.

7 The current upper limit of the sum of the neutrino masses is given by the Planck and baryon acoustic oscillation measurements as $\sum m_{v}<$ $0.12 \mathrm{eV}$ (95\% CL) [63], which corresponds to $m_{v} \sim 0.04 \mathrm{eV}$ in the degenerate case. Although it is ruled out, we also show the $n_{v}=3$ case in this paper to illustrate the $n_{v}$ dependence.
}

we assume that the $H$-loop contribution to the $\phi^{4}$ term as well as the field dependent masses of $\phi$ and $S$ coming from $H$ are negligibly small, which can be realized by taking $\lambda_{\phi H} \ll \lambda_{\phi S}$ and $\lambda_{S H} H^{\dagger} H \ll \lambda_{\phi S} \phi^{2}$. This potential can be rewritten at the scale $\mu=\mu_{*}$ where $\lambda_{\phi}$ vanishes as

$$
\begin{aligned}
V_{\text {eff }}= & \lambda_{H}\left(H^{\dagger} H-\frac{\lambda_{\phi H}}{\lambda_{H}} \phi^{2}\right)^{2}+\Delta V_{1-\text { loop }}\left(h, \mu_{*}\right) \\
& +\frac{\mu_{\phi}}{3 !} \phi^{3}+\frac{\mu_{\phi}^{2} \phi^{2}}{64 \pi^{2}}\left[\ln \frac{\mu_{\phi} \phi}{\mu_{*}^{2}}-\frac{1}{2}\right] \\
& +\frac{\lambda_{\phi S}^{2} \phi^{4}}{256 \pi^{2}}\left[\ln \frac{\lambda_{\phi S} \phi^{2}}{2 \mu_{*}^{2}}-\frac{1}{2}\right]-\frac{\lambda_{\phi H}^{2}}{\lambda_{H}} \phi^{4}
\end{aligned}
$$

In the following, we also assume that the $\mu_{\phi}^{2} \phi^{2}$ term is much smaller than the $\lambda_{\phi S}^{2} \phi^{4}$ term. ${ }^{8}$ Requiring the existence of the two saddle points at $\phi=0$ and $\phi=\phi_{\text {saddle }}(\mathrm{CP} 2-2),{ }^{9} \mu_{\phi}$ is determined to be

$\mu_{\phi}=\frac{\lambda_{\phi S}^{2}}{16 \pi^{2}} \phi_{\text {saddle }}, \quad$ with $\quad \phi_{\text {saddle }}=-\frac{1}{e} \sqrt{\frac{2}{\lambda_{\phi S}}} \mu_{*} e^{\frac{8 \pi^{2} \lambda_{\phi H}^{2}}{\lambda_{H} \lambda_{\phi S}^{2}}}$.

The vacuum expectation value (VEV) of $\phi,\langle\phi\rangle$, is then determined as

$\langle\phi\rangle=e^{W(1 / e)}\left(-e \phi_{\text {saddle }}\right)$,

where $W$ is the Lambert $W$ function. Using Eqs. (5) and (6), we obtain the relation between $\mu_{*}$ and $\langle\phi\rangle$ as

$\mu_{*}=\sqrt{\frac{\lambda_{\phi S}}{2}}\langle\phi\rangle \exp \left[-W\left(e^{-1}\right)-\frac{8 \pi^{2} \lambda_{\phi H}^{2}}{\lambda_{H} \lambda_{\phi S}^{2}}\right]$.

Now, it is clear that by looking at the first line of Eq. (4) the EW symmetry breaking is triggered by $\langle\phi\rangle$ :

$$
\frac{v}{\langle\phi\rangle}=\sqrt{\frac{\lambda_{\phi H}}{2 \lambda_{H}}},
$$

where $v \simeq 246 \mathrm{GeV}$. At the minimum, the squared masses of $\phi$ and $S$ are given by

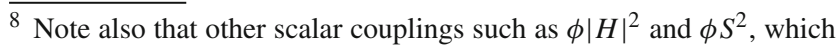
are set to zero by the MPP, are induced at the one-loop level as well. However, in the present analysis, the mixing between $H$ and $\phi$ is small, and since we are interested in $\langle S\rangle=0$, these effects are small.

${ }^{9}$ Note that this saddle point is nothing to do with the saddle point of the Higgs potential discussed in the next section.
} 
$m_{\phi}^{2} \sim-\frac{\lambda_{\phi H}}{2} v^{2}+\frac{1+W\left(e^{-1}\right)}{32 \pi^{2}} \lambda_{\phi S}^{2}\langle\phi\rangle^{2}$,

$m_{S}^{2}=\frac{\lambda_{S H}}{2} v^{2}+\frac{\lambda_{\phi S}}{2}\langle\phi\rangle^{2}$,

where we have neglected the mixing between $h$ and $\phi$. From the above discussion, the six parameters $\lambda, \lambda_{\phi}, \lambda_{\phi S}, \lambda_{S}, \lambda_{\phi H}$, and $\lambda_{S H}$ in the potential are reduced by fixing the two parameters, $v$ and the Higgs mass $125.1 \mathrm{GeV}$, and by taking $\lambda_{S}$ to be zero at the EW scale as aforementioned. The resultant three parameters are

$m_{S}, \quad \lambda_{S H}, \quad\langle\phi\rangle$,

where $\lambda_{\phi}$ has been replaced by the scale $\mu_{*}$ at which $\lambda_{\phi}$ vanishes, and further converted to $\langle\phi\rangle$ by Eq. (7).

The thermal relic abundance of $S$ is satisfied when [62]

$4 \lambda_{S H}^{2}+\lambda_{\phi S}^{2}=\left(\frac{m_{S}}{m_{\mathrm{th}}}\right)^{2}, \quad m_{\mathrm{th}}=1590 \pm 40 \mathrm{GeV}$

This relation provides a contour of $\langle\phi\rangle$ in the $m_{S}$ vs $\lambda_{S H}$ plane as shown in Fig. 2: the red, magenta, green, and blue curves correspond to $\langle\phi\rangle=2.5 \mathrm{TeV}, 3 \mathrm{TeV}, 4 \mathrm{TeV}$, and $10 \mathrm{TeV}$, respectively. We also show the other constraints on the model parameters in the figure: The purple, orange, cyan, and yellow shaded regions are excluded by the updated XENON1T result $[64],{ }^{10}$ the LHC results, DM relic abundance, and perturabativity bound, respectively [62]. We note that these bounds are insensitive to $M_{R}{ }^{11}$

In the following analyses of the critical Higgs inflation, we choose the parameters on the dotted line in Fig. 2 that is close to the perturbativity bound, to reduce the computational time. ${ }^{12}$ On the dotted line, there remains only single parameter in the scalar sector, and we choose it to be $\lambda_{S H}$. Therefore, there are two parameters $\lambda_{S H}$ and $M_{R}$ in total. We choose these parameters in such a way that the Higgs potential has a (near) saddle-point at high scale region. ${ }^{13}$

\footnotetext{
10 For the region with the DM mass larger than $1 \mathrm{TeV}$, we extrapolate the upper limit on the spin independent cross section for DM and nucleon scatterings.

$11 M_{R}$ might possibly affect the perturbativity bound via the RGE of $\lambda_{S}$ and $\lambda_{S H}$, but its effect is only through the small $\lambda_{H}$ coupling and is negligible.

12 When we vary $m_{S}$ from the dotted line for a fixed $\lambda_{S H}$, the coupling $\lambda_{\phi S}$ changes via Eq. (9), but the running of $\lambda$ and $\lambda_{H}$ do not depend on $\lambda_{\phi S}$ at the one-loop level (see Eq. (56)), and hence the high-scale Higgs potential is not altered drastically. Note also that, even though the dotted line is close to the perturbativity bound, $\lambda_{S H}$ (which contributes to running of $\lambda$ and $\lambda_{H}$ at one-loop) remains perturbative unlike $\lambda_{S}$, and hence the effective Higgs potential is reliably computed.

13 Without the right-handed neutrinos, the high-scale potential value tends to become too large to accommodate the observed value of the tensor-to-scalar ratio; see also Ref. [22].
}

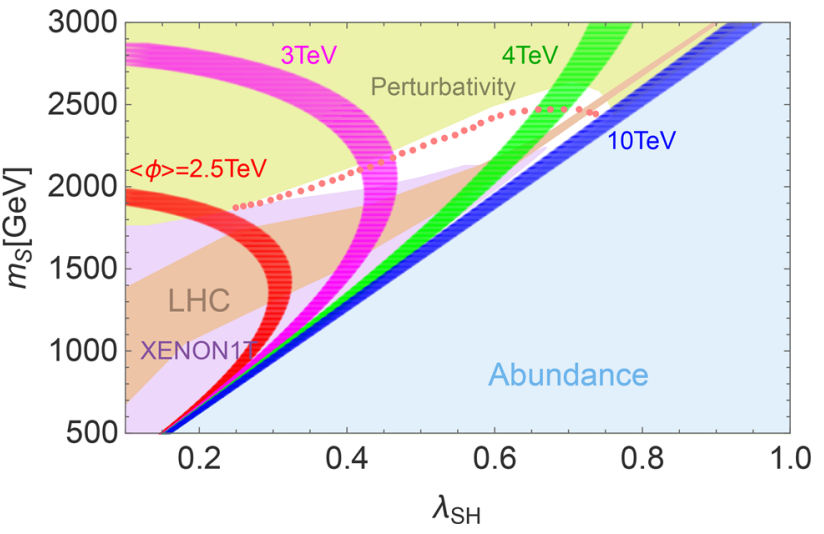

Fig. 2 The solid curves correspond to Eq. (11) for $\langle\phi\rangle=2.5 \mathrm{TeV}$ (red), $3 \mathrm{TeV}$ (magenta), $4 \mathrm{TeV}$ (green), and $10 \mathrm{TeV}$ (blue), with each width corresponding to the error of $m_{\text {th }}=1590 \pm 40 \mathrm{GeV}$. Shaded regions are respectively excluded by the XENON1T experiment (purple), the LHC data (orange), DM abundance (cyan), and the perturbativity bound (yellow). Regarding the perturbativity bound, the absence of Landau pole up to $\mu=10^{17} \mathrm{GeV}$ is imposed

\section{Saddle point of Higgs potential}

Now that the TeV-scale couplings are obtained, we extrapolate them towards high scales. In this section, we analyze the effective Higgs potential for large field values, and look for its saddle point in order to realize the critical Higgs inflation.

\subsection{Effective potential}

We calculate the one-loop effective Higgs potential improved by the two-loop RGEs presented in Appendix A. The oneloop effective potential for large $h$ in the $\overline{\mathrm{MS}}$ scheme in the Landau gauge is

$V=\frac{\lambda_{H}(\mu)}{4} \bar{h}^{4}+\Delta V_{1-\mathrm{loop}}(h, \mu)$,

where

$$
\begin{aligned}
\Delta V_{\text {1-loop }}(h, \mu)= & \frac{6 M_{W}^{4}(h)}{64 \pi^{2}}\left[\ln \left(\frac{M_{W}^{2}(h)}{\mu^{2}}\right)-\frac{5}{6}\right] \\
& +\frac{3 M_{Z}^{4}(h)}{64 \pi^{2}}\left[\ln \left(\frac{M_{Z}^{2}(h)}{\mu^{2}}\right)-\frac{5}{6}\right] \\
& -\frac{3 M_{t}^{4}(h)}{16 \pi^{2}}\left[\ln \left(\frac{M_{t}^{2}(h)}{\mu^{2}}\right)-\frac{3}{2}\right] \\
& -\sum_{i=1}^{3} \frac{M_{N_{i}}^{4}(h)}{32 \pi^{2}}\left[\ln \left(\frac{M_{N_{i}}^{2}(h)}{\mu^{2}}\right)-\frac{3}{2}\right]
\end{aligned}
$$




$$
\begin{aligned}
& +\frac{M_{S}^{4}(h)}{64 \pi^{2}}\left[\ln \left(\frac{M_{S}^{2}(h)}{\mu^{2}}\right)-\frac{3}{2}\right] \\
& +\frac{M_{\phi}^{4}(h)}{64 \pi^{2}}\left[\ln \left(\frac{M_{\phi}^{2}(h)}{\mu^{2}}\right)-\frac{3}{2}\right],
\end{aligned}
$$

in which the effective masses are

$$
M_{W}(h)=\frac{g_{2} h}{2}, \quad M_{Z}(h)=\frac{\sqrt{g_{2}^{2}+g_{Y}^{2}}}{2} h, \quad M_{t}(h)=\frac{y_{t} h}{\sqrt{2}},
$$

$M_{N_{i}}(h)=\frac{M_{R}}{2}\left(1+\sqrt{1+\frac{2 y_{v i}^{2} h^{2}}{M_{R}^{2}}}\right), \quad M_{S}^{2}(h)=\frac{\lambda_{S H}}{2} h^{2}$,

$M_{\phi}^{2}(h)=\frac{\lambda_{\phi H}}{2} h^{2}$,

and

$$
\bar{h}:=h e^{\Gamma(\mu)}=h \exp \left(\int_{0}^{\ln \mu / M_{t}} d s \gamma_{H}\right)
$$

is the Higgs field with field renormalization. See Eq. (64) in Appendix A for the one-loop result of $\gamma_{H}$. We here represent $N_{i}$ as the mass eigenstates for the heavy Majonara neutrinos. We neglect the contributions from the leptons and quarks other than the top quark because their Yukawa couplings are small. We also neglect the loops of the Higgs and the NG bosons because $\lambda_{H}$ becomes small at high scales.

In the following, we match two theories with and without right-handed neutrinos around $\mu=M_{R}$, to obtain the threshold correction. We expand the one-loop correction from heavy neutrinos by $h$ as

$$
\begin{aligned}
& -\sum_{i=1}^{3} \frac{M_{N_{i}}^{4}(h)}{32 \pi^{2}}\left[\ln \left(\frac{M_{N_{i}}^{2}(h)}{\mu^{2}}\right)-\frac{3}{2}\right] \\
& =-\frac{1}{32 \pi^{2}}\left[\frac{3 M_{R}^{4}}{2}\left(-3+4 \ln \left(\frac{M_{R}}{\mu}\right)\right)\right. \\
& +2 h^{2} M_{R}^{2} n_{v} y_{v}^{2}\left(-1+2 \ln \left(\frac{M_{R}}{\mu}\right)\right) \\
& \left.+\frac{h^{4}}{2} n_{v} y_{v}^{4}\left(1+2 \ln \left(\frac{M_{R}}{\mu}\right)\right)+\cdots\right],
\end{aligned}
$$

where the coefficient of $h^{4}$ corresponds to the threshold correction to $\lambda_{H}$ below $M_{R}[65-67]^{14}$ :

$\Delta \lambda_{H}^{(R)}:=-\frac{n_{v} y_{v}^{4}}{16 \pi^{2}}\left(1+2 \ln \left(\frac{M_{R}}{\mu}\right)\right)$.

The term containing $\ln \left(M_{R} / \mu\right)$ leads to the subtractions of $y_{v}^{4}$ term from the beta function. The same analysis can be also applied to the field renormalization as

$$
\begin{aligned}
& \frac{1}{2} h \square h\left[1-\frac{2}{32 \pi^{2}} \sum_{i=1}^{3} y_{v i} \ln \left(\frac{M_{N_{i}}^{2}(h)}{\mu^{2}}\right)\right] \\
& \simeq \frac{1}{2} h \square h\left[1-\frac{1}{8 \pi^{2}} n_{v} y_{v}^{2} \ln \left(\frac{M_{R}}{\mu}\right)+\cdots\right],
\end{aligned}
$$

from which we can see that the canonically normalized Higgs field $h_{c}$ in the low-energy theory is given by

$h_{c}=h\left[1-\frac{1}{8 \pi^{2}} n_{\nu} y_{v}^{2} \ln \left(\frac{M_{R}}{\mu}\right)\right]^{1 / 2}=: h Z_{R}$.

This also leads to the redefinition of the quartic coupling. By combing both of them, the Higgs quartic coupling below $\mu=M_{R}$ is ${ }^{15}$

$$
\begin{aligned}
\lambda: & =\lambda_{H} Z_{R}^{-4}+\Delta \lambda_{H}^{(R)} \\
= & \lambda_{H}\left(1-\frac{4 n_{v}}{16 \pi^{2}} y_{v}^{2} \ln \left(\frac{\mu}{M_{R}}\right)\right)+\frac{n_{v} y_{v}^{4}}{16 \pi^{2}} \\
& \times\left(-1+2 \ln \left(\frac{\mu}{M_{R}}\right)\right) .
\end{aligned}
$$

One can easily check that contributions from heavy neutrinos cancel out in the beta function $d \lambda / d \ln \mu$.

In Fig. 3, we show the RG runnings of $\lambda$ and $\lambda_{H}$, where the upper left (right) panel corresponds to the normal (inverted) hierarchy case and the lower panel corresponds to the degenerate case. The different colors correspond to the different values of $\lambda_{S H}\left(M_{t}\right)$, and the top pole mass $m_{t}^{\text {pole }}$ and $M_{R}$ are fixed at $m_{t}^{\text {pole }}=172.4 \mathrm{GeV}$ [5] and $4 \times 10^{14} \mathrm{GeV}$, respectively. As explained above, other parameters, $\langle\phi\rangle$ and $m_{S}$, are fixed as functions of $\lambda_{S H}$ on the dotted line in Fig. 2 by the thermal relic abundance of $S$ to explain $\Omega_{\mathrm{DM}} h^{2} \sim 0.12$.

14 The coefficient of $h^{2}$ in Eq. (17) gives the threshold correction to the Higgs mass-squared parameter in the low-energy theory. At the $\mathrm{CP}$ 2-2, the mass-squared parameter including this correction is tuned to zero, based on the MPP. See Refs. [61,62] for the detailed discussion.

15 If we want, we may take into account the threshold correction of $S$ too: $\Delta \lambda_{H}^{(S)}=\frac{\lambda_{S H}^{2}}{16 \pi^{2}}\left(\frac{1}{6}+\frac{1}{2} \ln \left(\frac{m_{S}}{\mu}\right)\right)$. This contribution is minor for our analysis and we neglect it hereafter. 

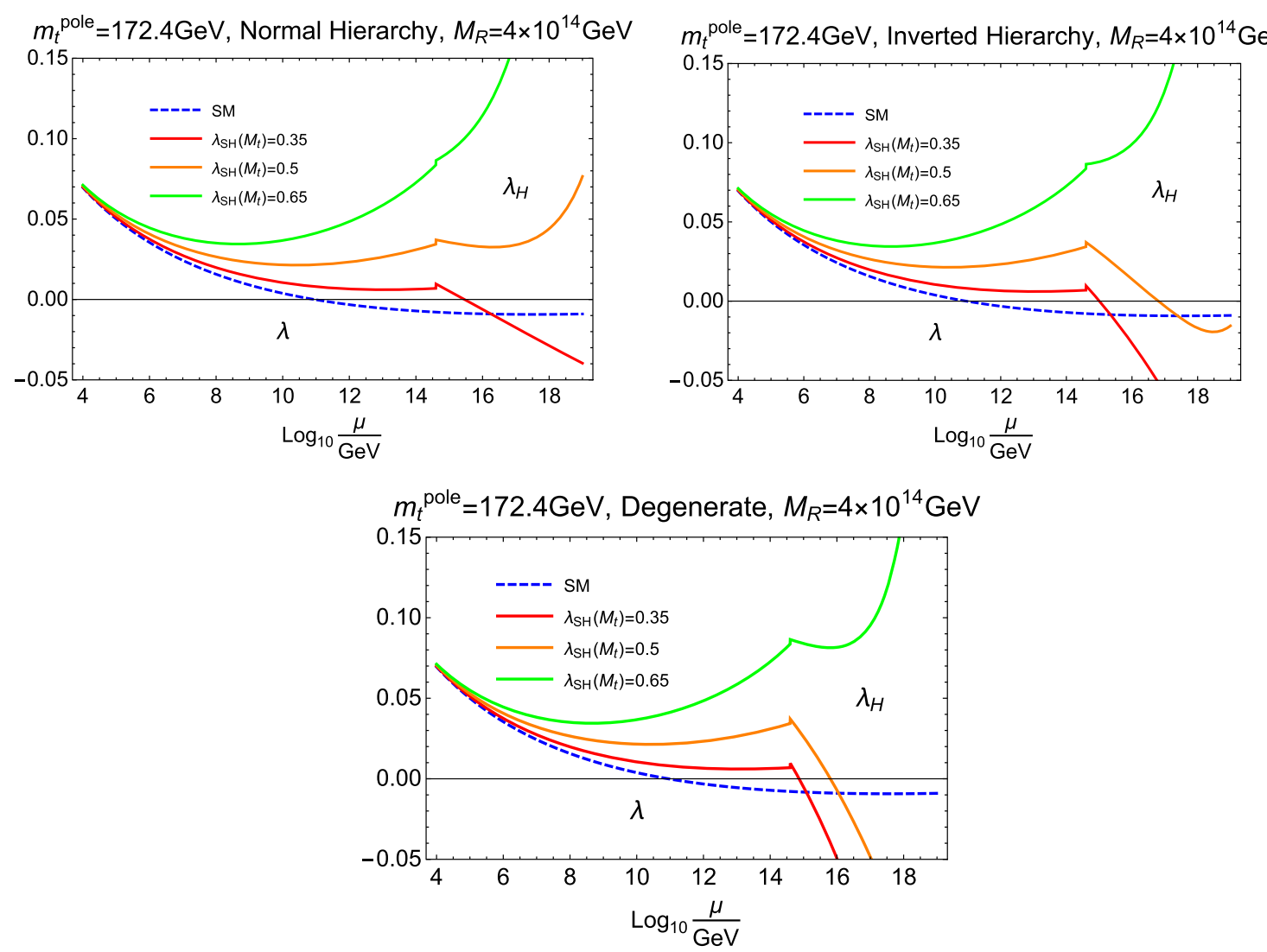

Fig. 3 The RG runnings of $\lambda$ and $\lambda_{H}$. Upper left (right) corresponds to the case of normal (inverted) hierarchy and lower panel corresponds to the degenerate case. Here, the different colors correspond to the different values of $\lambda_{S H}\left(M_{t}\right)$

\subsection{Saddle point}

In the following, we rewrite the one-loop Higgs effective potential (12) as

$V=\frac{\lambda_{\mathrm{eff}}(h, \mu)}{4} h^{4}$

where

$\lambda_{\text {eff }}(h, \mu)=\left[\lambda_{H}(\mu) e^{4 \Gamma(\mu)}+4 \frac{\Delta V_{\text {1-loop }}(h, \mu)}{h^{4}}\right]$.

Note that $\lambda_{\text {eff }}(h, \mu)$ is independent of the renormalization point $\mu$ if we take all order loop corrections into account. For practical purpose with finite-loop, it better approximates by choosing $\mu \sim h$.

In the SM without the right-handed neutrinos, it is known that $\lambda_{\text {eff }}(h, h)$ in general takes a minimum value $\lambda_{\text {min }}$ at $h=h_{\min }\left(\simeq 10^{18} \mathrm{GeV}\right)$; see the blue dashed line in Fig. 3 . Furthermore, by tuning the top mass, we can realize a saddle point $V^{\prime}=V^{\prime \prime}=0$ at $h=h_{s}\left(\simeq h_{\min }\right)$ [1]. (This tuning may be done within the $1.4 \sigma$ experimental bound as stressed in Introduction.) However, this saddle-point itself is not sufficient to achieve a viable saddle-point inflation because the resultant CMB fluctuations become too large $[42,68,69]$. By introducing the non-minimal coupling $\xi|H|^{2} R$, a successful inflation can be achieved around the (near) saddle-point even when $\xi \sim 10[1,19,20]$. This is called the critical Higgs inflation.

In this paper, we pursue the critical Higgs inflation in our model at the CP 2-2. The detailed analysis will be presented in the next section. In the remaining of this section, we look for the saddle point in our model. When we take into account the right-handed neutrinos, they first lower $\lambda_{H}$ above $M_{R}$, and at higher scales, the extra scalar couplings become large and their contributions raise $\lambda_{H}$ again. As a result, we may have a minimum for $\lambda_{H}$ at a high scale as can be seen in Fig 3, e.g. with the orange curve for the normal and inverted hierarchy cases, and green for the degenerate case.

In general, the condition to have a saddle point is

$$
\begin{aligned}
V^{\prime} & =V^{\prime \prime}=0 \Leftrightarrow \lambda_{\text {eff }}(h, h)+\frac{1}{4} \frac{d \lambda_{\text {eff }}(h, h)}{d \ln h} \\
& =12 \lambda_{\text {eff }}(h, h)+8 \frac{d \lambda_{\text {eff }}(h, h)}{d \ln h}+\frac{d^{2} \lambda_{\text {eff }}(h, h)}{d(\ln h)^{2}}=0 .
\end{aligned}
$$


Again, we call the position of saddle-point $h_{s}$. As said in the last of Sect. 2, there is only single parameter $\lambda_{S H}$ in the scalar sector. For each given $\lambda_{S H}$, we numerically solve for $M_{R}$ that achieves the saddle-point condition (24), and obtain its position $h_{s}$.

In the upper left panel in Fig. 4, we show the Higgs potential in the case of the normal hierarchy where the differently colored contours correspond to the different values of $\lambda_{S H}$. Here, the Higgs field $h$ is normalized by $h_{s}$. In the upper right panel, we plot the value of $M_{R}$ as a function of $\lambda_{S H}$ that is determined by the saddle point condition (24). In the lower left panel, we show $h_{S}$ as a function of $\lambda_{S H}$ where blue and red correspond to the normal and inverted hierarchy cases, respectively, while green to degenerate. One can see that $h_{s}$ has a strong dependence on $\lambda_{S H}$, which causes the strong dependence of the value of the potential around the saddle point on $\lambda_{S H}$ in the upper left panel.

The above numerical result can be fitted by an analytic formula as follows. Around the minimum $h=h_{\min }$ giving the minimal value of $\lambda_{\text {eff }}$, we may always approximate as [1]

$\lambda_{\text {eff }}(h, h)=\lambda_{\text {min }}+\frac{b}{2}\left(\ln \frac{h}{h_{\text {min }}}\right)^{2}$.

Within this approximation, we see that the potential has a saddle point $V^{\prime}=V^{\prime \prime}=0$ at $h=h_{s}$ when and only when $\lambda_{\min }$ is tuned to a critical value $\lambda_{\min }=\lambda_{c}$ where

$h_{s}=e^{-1 / 4} h_{\min }$,

$\lambda_{c}=\frac{b}{32}$.

That is,

$\lambda_{\text {eff }}(h)=\frac{b}{32}\left[1+16\left(\ln \left(\frac{h}{h_{s} e^{1 / 4}}\right)\right)^{2}\right]$.

We fit $b$ and $h_{s}$ from the numerical analysis above. As a consistency check, we also plot the Higgs potential whose effective coupling is replaced by the analytical one (28) in the upper left panel in Fig. 4 by dashed black lines. One can actually see that the analytical ones well approximate the effective Higgs potential around the saddle point. In the next section, we will use Eq. (25) with $\lambda_{\min }$ close to $\lambda_{c}$, as in Eq. (50), to study the critical Higgs inflation. In particular, the minimum value $\lambda_{c}$ is important because it also determines, along with $h_{s}$, the value of the potential during the inflation. Qualitatively, small $\lambda_{c}$ will turn out to be favorable to realize successful inflation with small $\xi \lesssim 100$.

In the lower right panel in Fig. 4, we show the numerical calculations of $\lambda_{c}$ as a function of $\lambda_{S H}$. From this plot, one can see that $\lambda_{c}$ is a monotonically increasing function of $\lambda_{S H}$ and its order of magnitude is $10^{-5}$ around $\lambda_{S H} \sim 0.3$. This corresponds to $b=\mathcal{O}\left(10^{-4}\right)$ by Eq. (27). We will see that the region with small $\lambda_{S H}$ is preferred to realize a successful inflation for small $\xi .{ }^{16}$ Therefore, in the inflationary analysis, we will show the results in the region $\lambda_{S H} \lesssim 0.3$.

\section{Critical Higgs inflation}

In this section, we study the critical Higgs inflation of our model at the CP 2-2. We take full advantage of the saddle point of the Higgs potential. The existence of the saddle point makes it easy to obtain the sufficient number of e-foldings $N$ even when $\xi \lesssim 100$; contrary to the conventional case, the tensor-to-scalar ratio $r$ does not have to be related to $N$ as $r \sim 1 / N^{2}$, and it can be sizable $\sim 0.05$. In Sect. 5.1, we will show our numerical calculations of CMB observables.

Here we comment on the other fields. During the critical Higgs inflation, the Higgs potential is flat, while other scalar fields have large masses due to the couplings $\lambda_{\phi H}$ and $\lambda_{S H}$ with the large Higgs field, so they do not play a role in the inflation analysis. As for the renormalization scale, since we are considering the case where only the Higgs field is large, we can consider the Higgs field $h$ as a renormalization point and use the single-scale renormalization group.

\subsection{Higgs inflation at classical level}

We first review the Higgs inflation with non-minimal coupling $\xi h^{2} R$ at the classical level $[28,70]$. We start with the Jordan-frame action

$$
\begin{aligned}
S_{\mathrm{cl}}= & \frac{M_{\mathrm{P}}^{2}}{2} \int d^{4} x \sqrt{-g_{J}} \Omega^{2}(h) R_{J}-\int d^{4} x \sqrt{-g_{J}} \\
& \times\left[\frac{1}{2}(\partial h)^{2}+V_{\mathrm{cl}}(h)\right],
\end{aligned}
$$

where we have truncated the potential and Weyl factor at the quartic and quadratic orders, respectively:

$$
V_{\mathrm{cl}}(h)=\frac{\lambda_{\mathrm{cl}}}{4} h^{4}, \quad \Omega^{2}(h)=1+\xi \frac{h^{2}}{M_{\mathrm{P}}^{2}} .
$$

By performing the following redefinition of the metric,

$g_{\mu \nu}=\Omega^{2} g_{J \mu \nu}$,

we obtain

$R_{J}=\Omega^{2}\left[R+3 \square \ln \Omega^{2}-\frac{3}{2} g^{\mu \nu}\left(\partial_{\mu} \ln \Omega^{2}\right)\left(\partial_{\nu} \ln \Omega^{2}\right)\right]$.

\footnotetext{
16 Higgs inflation with $\xi=\mathcal{O}\left(10^{4}\right)[28,70]$ is always possible at the expense of the small cutoff scale $M_{\mathrm{P}} / \xi$.
} 

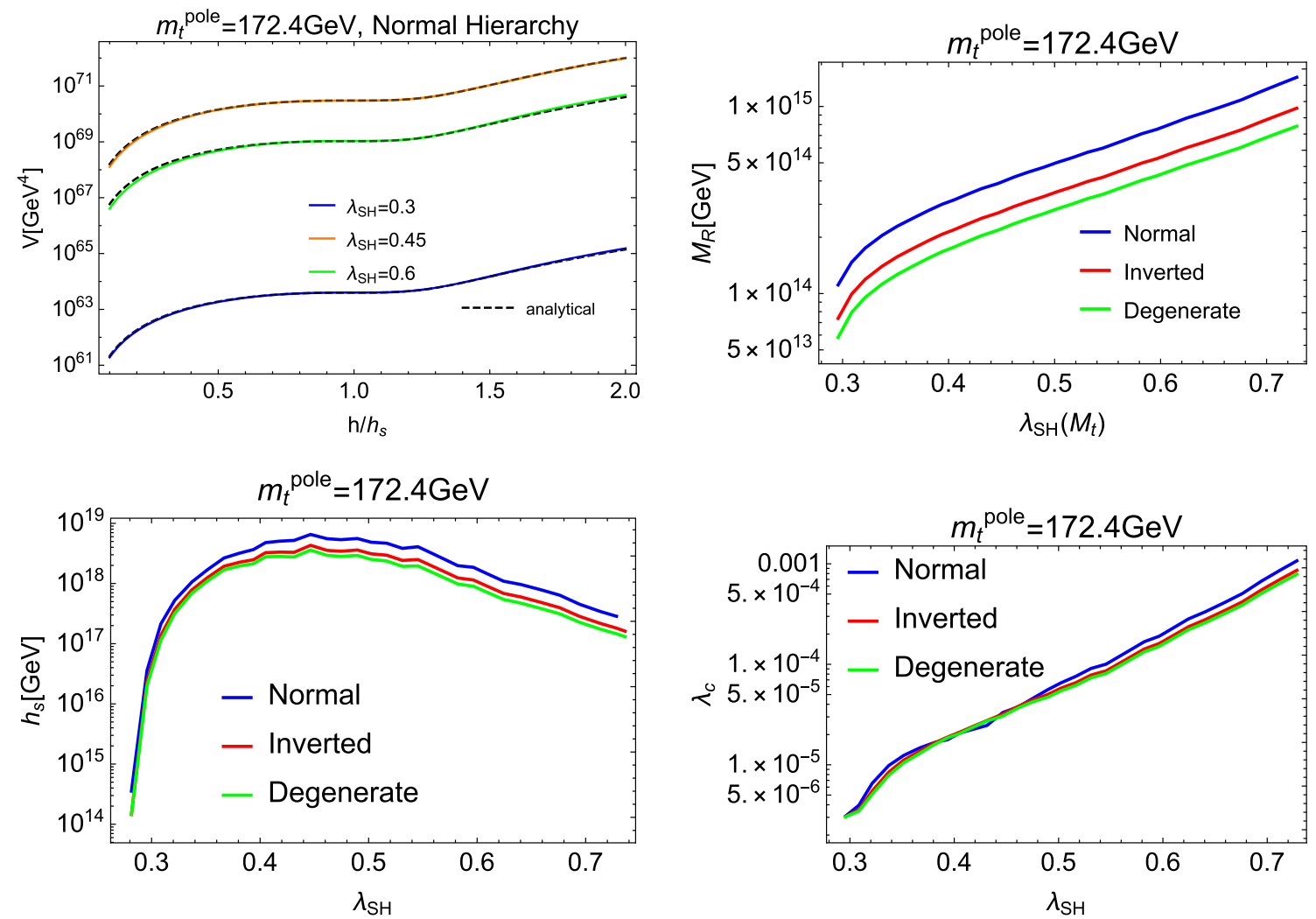

Fig. 4 Upper Left: the one-loop effective Higgs potential in the normal hierarchy case where the different colors correspond to the different values of $\lambda_{S H}\left(M_{t}\right)$. Here, dashed black curves represent the Higgs potential whose quartic coupling is replaced by the analytical one (28). Upper Right: the relation between $M_{R}$ and $\lambda_{S H}\left(M_{t}\right)$ determined by the saddle

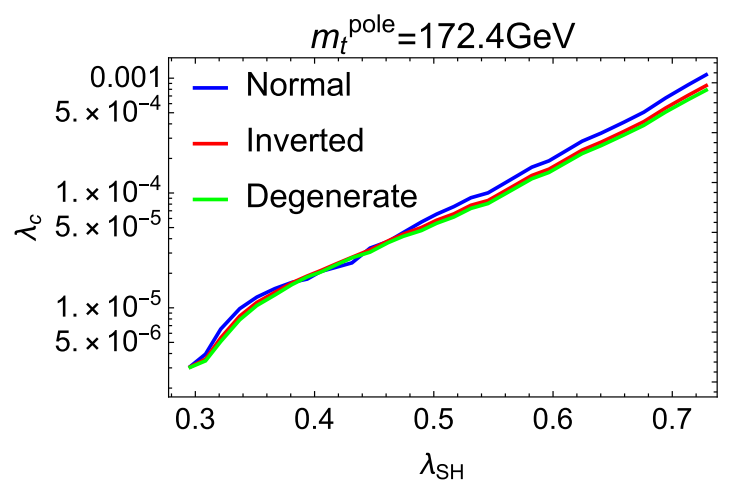

point condition (24). Lower Left: the saddle point $h_{S}$ as a function of $\lambda_{S H}\left(M_{t}\right)$, where blue and red correspond to the normal and inverted hierarchy, while green to degenerate. Lower Left: the minimum value of $\lambda_{H}$ as a function of $\lambda_{S H}\left(M_{t}\right)$

Then the Einstein-frame action becomes

$$
\begin{aligned}
S_{\mathrm{cl}}= & \int d^{4} x \sqrt{-g}\left(\frac{1}{2} M_{\mathrm{P}}^{2} R-\frac{1}{2 \Omega^{2}}(\partial h)^{2}\right. \\
& \left.-\frac{3}{4} M_{\mathrm{P}}^{2}\left(\partial_{\mu} \ln \Omega^{2}\right)^{2}-U_{\mathrm{cl}}(h)+\cdots\right),
\end{aligned}
$$

where

$U_{\mathrm{cl}}(h)=\frac{V_{\mathrm{cl}}(h)}{\Omega^{4}(h)}$

is the potential in the Einstein frame. Since $V_{\mathrm{cl}}$ is quartic, the following relation holds

Therefore for large $h$, the Einstein-frame potential $U_{\mathrm{cl}}(h)$ becomes constant:

$U_{\mathrm{cl}}(h) \rightarrow V_{\mathrm{cl}}\left(\frac{M_{\mathrm{P}}}{\sqrt{\xi}}\right)=\frac{\lambda_{\mathrm{cl}} M_{\mathrm{P}}^{4}}{4 \xi^{2}}$.

This flat potential is used in the Higgs inflation.

The relation between the canonically normalized Einsteinframe field $\chi$ and the Jordan-frame field $h$ is given by

$\frac{d \chi}{d h}=\frac{\sqrt{\Omega^{2}+6 \xi^{2} h^{2} / M_{\mathrm{P}}^{2}}}{\Omega^{2}}$.

Under the slow-roll approximation, we obtain

$U_{\mathrm{cl}}(h)=\frac{\lambda_{\mathrm{cl}}}{4}\left(\frac{h}{\Omega(h)}\right)^{4}=V_{\mathrm{cl}}\left(\frac{h}{\Omega(h)}\right)$.

In the $h \rightarrow \infty$ limit, we have

$\frac{h}{\Omega(h)} \rightarrow \frac{M_{\mathrm{P}}}{\sqrt{\xi}}$

$\frac{\lambda_{\mathrm{cl}}}{\xi^{2}} \simeq 6.0 \times\left(\frac{50}{N}\right)^{2} \times 10^{-10}$

to fit $A_{s}=U /\left(24 \pi^{2} M_{\mathrm{P}}^{4} \varepsilon_{V}\right)$ to the observed value $2.1 \times 10^{-9}$ at the $e$-folding $N$; see Appendix C. We see that the typical $\mathrm{SM}$ value at low energy $\lambda_{\mathrm{cl}} \sim 0.1$ requires large value of $\xi \sim 10^{5}$. 


\subsection{Higgs inflation including radiative correction}

At the quantum level in the flat spacetime, we promote $V_{\mathrm{cl}}(h)$ to the effective potential

$V(h)=V_{\text {tree }}(h, \mu)+\Delta V_{\text {loop }}(h, \mu)$,

where $V_{\text {tree }}$ is the tree-level potential including the field renormalization and $\Delta V_{\text {loop }}$ is the loop correction; see Eq. (12) for the 1-loop approximation. In this paper, we employ the Einstein-frame effective potential on the so-called Prescription I,

$U(h)=\frac{\lambda_{H}(\mu) e^{4 \Gamma(\mu)} h^{4}}{4 \Omega^{4}(h)}+\Delta U_{\text {loop }}(h, \mu)$,

where $\Delta U_{\text {loop }}$ is obtained from $\Delta V_{\text {loop }}$ in Eq. (40) by replacing all the effective masses $M_{\Psi}(h)$ with $\frac{M_{\Psi}(h)}{\Omega}$ for $\Psi=W, Z$, $t, v i, S$, and $\phi$ :

$\Delta U_{\text {loop }}(h, \mu)=\left.\Delta V_{\text {loop }}(h, \mu)\right|_{M_{\Psi}(h) \rightarrow \frac{M_{\Psi}(h)}{\Omega}}$.

See Ref. [71] for the meaning of this prescription as well as ambiguity due to indeterminacy of non-renormalizable terms. Because the potential should not depend on $\mu$ (if one takes all order corrections into account), we may replace $\mu$ by $\mu / \Omega^{17}$ :

$$
\begin{aligned}
U(h) & =\frac{\lambda_{H}\left(\frac{\mu}{\Omega}\right) e^{4 \Gamma\left(\frac{\mu}{\Omega}\right)} h^{4}}{4 \Omega^{4}}+\left.\Delta V_{\text {loop }}\left(h, \frac{\mu}{\Omega}\right)\right|_{M_{\Psi}(h) \rightarrow \frac{M_{\Psi}(h)}{\Omega}} \\
& =\frac{\lambda_{H}\left(\frac{\mu}{\Omega}\right) e^{4 \Gamma\left(\frac{\mu}{\Omega}\right)} h^{4}}{4 \Omega^{4}}+\frac{1}{\Omega^{4}} \Delta V_{\text {loop }}(h, \mu) .
\end{aligned}
$$

When we truncate the loop correction at the 1-loop order, we can obtain better approximation by choosing $\mu$ to be around $h$ to minimize the higher loop corrections:

$U(h)=\frac{1}{\Omega^{4}}\left(\frac{\lambda_{H}\left(\frac{h}{\Omega}\right)}{4} e^{4 \Gamma\left(\frac{h}{\Omega}\right)} h^{4}+\Delta V_{1-\text { loop }}(h, h)\right)$.

This is the expression we employ in the following. When $y_{v i} h$ is sufficiently larger than $M_{R}$, all the effective masses are proportional to $h$, therefore $\Delta V_{1 \text {-loop }}(h, h)$ is proportional to $h^{4}$ whose coefficient is independent of $h$ (up to higher order corrections), and hence we obtain

$U(h)=V\left(\frac{h}{\Omega}\right)$

17 In the second line of Eq. (43), we have assumed that the $\Omega$ dependence in $\Delta V_{\text {loop }}(h, \mu / \Omega)$ disappears by the replacement $M_{\psi}(h) \rightarrow$ $M_{\psi}(h) / \Omega$. At one loop level, we can easily check this from Eq. (13).
Thus, after taking into account the quantum corrections, the same relation still holds as Eq. (35) on Prescription I, and again the Einstein-frame potential (45) becomes constant for $\xi h^{2} / M_{\mathrm{P}}^{2} \gg 1$ :

$$
\begin{aligned}
U(h)= & \frac{\lambda_{\mathrm{eff}}\left(\frac{h}{\Omega(h)}, \frac{h}{\Omega(h)}\right)}{4}\left(\frac{h}{\Omega(h)}\right)^{4} \rightarrow \frac{\lambda_{\mathrm{eff}}\left(\frac{M_{\mathrm{P}}}{\sqrt{\xi}}, \frac{M_{\mathrm{P}}}{\sqrt{\xi}}\right)}{4} \\
& \times\left(\frac{M_{\mathrm{P}}}{\sqrt{\xi}}\right)^{4} .
\end{aligned}
$$

Unless $\lambda_{\text {eff }}\left(\frac{M_{\mathrm{P}}}{\sqrt{\xi}}, \frac{M_{\mathrm{P}}}{\sqrt{\xi}}\right)$ is particularly small, we still need large $\xi$ to fit $A_{s}$. In fact in the SM, it is known that $\lambda_{\text {eff }}(h, h)$ becomes small around $h \sim M_{\mathrm{P}}$, which is an essential ingredient in the critical Higgs inflation.

\subsection{Critical Higgs inflation}

As can be seen from Eq. (45), when $V(h)$ has a saddle point at $h=h_{s}, U(h)$ also has a saddle point at $h=\tilde{h}_{s}$, with $\tilde{h}_{s}$ being determined by $\frac{\tilde{h}_{s}}{\Omega\left(\tilde{h}_{s}\right)}=h_{s}$ :

$\tilde{h}_{s}=\frac{h_{s}}{1-c_{s}^{2}}$

where we have introduced

$c_{s}:=h_{s} \frac{\sqrt{\xi}}{M_{\mathrm{P}}}$

This parameter is the ratio of $h_{s}$ to $M_{\mathrm{P}} / \sqrt{\xi}$; the latter is the typical value of $h$ above which the conformal factor $\Omega(h)$ starts to deviate from unity. One can see that $\tilde{h}_{s}$ approaches infinity as $c_{S} \nearrow 1$, which means that the small region around the saddle point $h=h_{s}$ is widely stretched, and allows a sufficient $e$-folding.

In the critical Higgs inflation, we assume that the highscale Higgs potential is close to a one having a saddle point, namely, $\lambda_{\min }$ in Eq. (25) is close to $\lambda_{c}$ given by Eq. (27):

$\lambda_{\min }=(1+\delta) \lambda_{c}$,

where we have parametrized the deviation from the saddlepoint criticality by $\delta$. Then the flat-space effective potential becomes

$V(h)=\frac{\lambda_{\mathrm{eff}}(h, h)}{4} h^{4}=\frac{\lambda_{c}}{4}\left[1+\delta+16\left(\ln \left(\frac{h}{h_{s} e^{1 / 4}}\right)\right)^{2}\right] h^{4}$ 


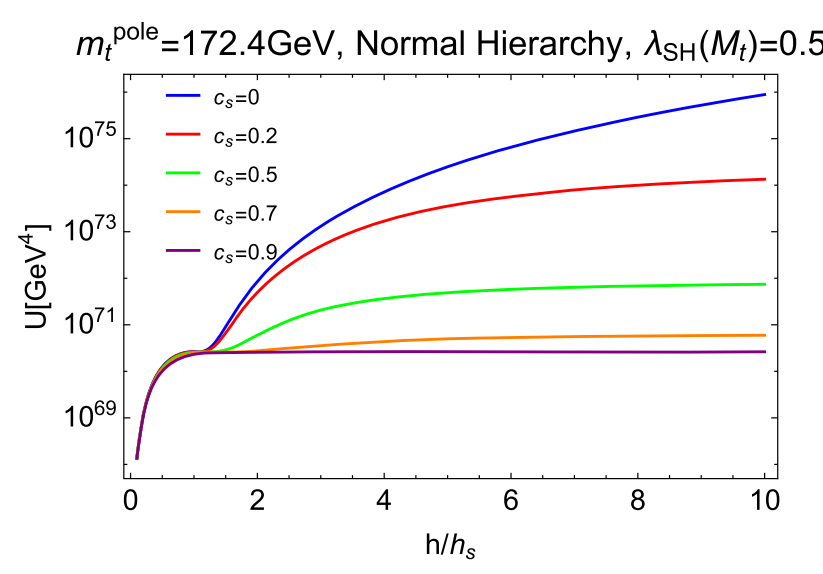

Fig. 5 The Higgs potential in the Einstein frame

Using Eqs. (45) and (48), we see that $U$ approaches the constant value in the $h \rightarrow \infty$ limit

$U(h) \rightarrow \frac{\lambda_{c}}{4}\left[1+\delta+\left(1+4 \ln c_{s}\right)^{2}\right] \frac{M_{\mathrm{P}}^{4}}{\xi^{2}}$,

which determines the value of the potential during the inflation. In this paper, we will focus on $c_{s} \leq 1$ and take full advantage of the saddle point of the Higgs potential.

In Fig. 5, we show the Higgs potential in the Einstein frame where different colors correspond to different values of $c_{S}$. Here, we show the the normal hierarchy case with $\lambda_{S H}\left(M_{t}\right)=0.5$ for illustration.

One can see that the region around the saddle point $h=h_{s}$ is more and more stretched as we increase $c_{s}$ toward unity. Therefore, for a given $c_{s} \lesssim 1$, we may always fit the $e$-folding $N$ around $h=h_{s} \Omega$ by tuning $\delta .^{18}$

\section{Prediction on inflationary observables}

Here, we analyze the prediction on inflationary observables as we vary the parameters in the model. So far, we have three free parameters: $\lambda_{S H}, M_{R}$, and $\xi$. Recall that the scalar sector has only one parameter $\lambda_{S H}$ in our analysis on the red dotted line in Fig. 2.

On the other hand, we have seen that the Jordan-frame potential can be parametrized near the saddle-point criticality by $\lambda_{c}, \delta$, and $h_{s}$ as Eq. (50). Here, these three parameters are functions of the model parameters $\lambda_{S H}$ and $M_{R}$.

In order to sweep the parameters near the saddle point, we use the results of Sect. 3.2: For each $\lambda_{S H}$, we find the value of $M_{R}$ that gives the saddle-point criticality, $\delta=0$, as well as the corresponding parameters $h_{s}$ and $\lambda_{c}$. See the upper-right,

\footnotetext{
$\overline{18}$ The tuning of $\lambda_{\min }$ to $\lambda_{c}$ is favored by the maximum entropy principle because the more the space is expanded by the inflation, the more the total entropy emerges $[10,14]$.
}

lower-left, and lower-right panels in Fig. 4 for $M_{R}, h_{s}$, and $\lambda_{c}$, respectively. For a given $\lambda_{S H}$, as we slightly change $M_{R}$ from the critical value, in general all of the parameters $\lambda_{c}$, $h_{s}$, and $\delta$ are modified. Here, we neglect the change of $\lambda_{c}$ and $h_{s}$, and take into account the effect of non-zero $\delta$.

Now we take into account the non-minimal coupling $\xi$. Among three parameters $\xi, \lambda_{S H}, \delta$, the last one is traded with $e$-folding $N$ using Eq. (66). The observables $n_{s}$ and $r$ are functions of $\xi$ and $\lambda_{S H}$ once $e$-folding $N$ is fixed by $\delta$.

\subsection{Results}

In Fig. 6, we show the values of $\xi, c_{S}$, and $\lambda_{S H}$ in the $n_{s}-r$ plane for $N=60$ with the central value $m_{t}^{\text {pole }}=172.4$ GeV: The upper left (right) panel corresponds to the normal (inverted) hierarchy case, and the lower panel to the degenerate case. The values of $\xi$ and $c_{s}$ are shown by the solid and dashed lines, respectively, while $\lambda_{S H}$ by the numbers on the solid line. The dark (light) blue region is allowed by the combined analysis of Planck 2018 at the 65\% (95\%) CL. From these results, one can see that our model at the CP 2-2 is consistent with the current $\mathrm{CMB}$ observations even when $\xi=25$. The smaller the $r$, the larger the required value of $\xi$ : If the upper bound becomes $r<0.04$ (0.02), we need $\xi \gtrsim 30$ (40).

We see that we typically have $\lambda_{S H} \sim 0.32$, which corresponds to the large Majorana mass as

$5 \times 10^{13} \mathrm{GeV} \lesssim M_{R} \lesssim 2 \times 10^{14} \mathrm{GeV}$

from the upper right panel in Fig. 4.

In Fig. 7, we also show $d n_{s} / d \ln k$ vs $n_{s}$, where $c_{s}$ and $\xi$ are again shown as in Fig. 6. In this case, the observational error of $d n_{s} / d \ln k$ is still too large to constrain the inflation potential.

\section{Summary and discussion}

Motivated by various fundamental issues in particle physics and cosmology, we have discussed the minimal model that can explain EW scale, neutrino masses, DM, and successful inflation at the same time. The model adds right-handed neutrinos to the two-scalar model in Refs. [61,62], which has been proposed to explain the origin of EW scale and DM. These two scalar fields give a minimal setup to realize an analogue of the CW mechanism. Assuming the $Z_{2}$ symmetry of a scalar, $S \rightarrow-S$, it can be a candidate for DM, similarly to the Higgs-portal scalar DM model. Neutrino masses are naturally explained by the seesaw mechanism.

In this paper, we have analyzed RGEs, calculated the effective Higgs potential, and studied the critical Higgs infla- 

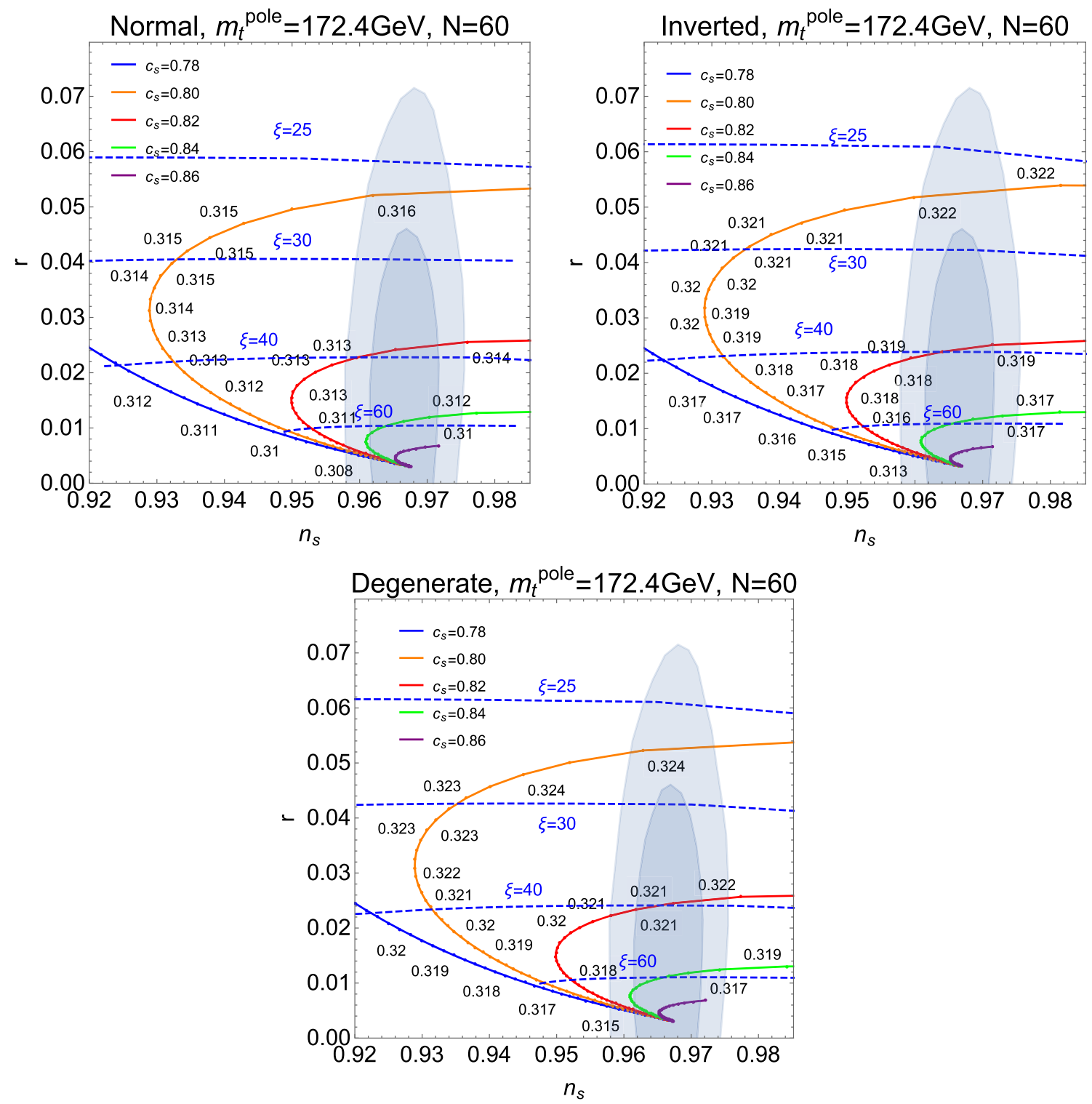

Fig. 6 Upper Left (Right): $r$ vs $n_{S}$ in the case of the normal (inverted) hierarchy where $c(\xi)$ is fixed on each solid (dashed) curves and $\lambda_{S H}\left(M_{t}\right)$ is varied. The blue regions correspond to the allowed regions by Planck 2018. Lower: the degenerate case

tion that uses the (near) saddle point of the Higgs potential at a high scale. The new scalar coupling $\lambda_{S H}$ between the SM Higgs and DM $S$ can stabilize the Higgs potential even if the top mass is current center value $m_{t}=172.4 \mathrm{GeV}$. In our model, it is possible to maintain the existence of saddle point by the neutrino Yukawa coupling $y_{v}$, and the saddle point condition relates the parameters of the model as is shown in Fig. 4.

By utilizing the saddle point of the Higgs potential, we have found that it is possible to realize successful inflation even for $\xi \sim 25$ within the parameter space where all the necessary requirements are satisfied. As a result, we obtain $\lambda_{S H} \simeq 0.32$ and $\langle\phi\rangle \simeq 2.7 \mathrm{TeV}$, which correspondingly lead to the dark matter mass $m_{S} \sim 2.0 \mathrm{TeV}$, its spin-independent cross section $1.8 \times 10^{-9} \mathrm{pb}$, and the mass of additional neutral scalar $m_{H} \sim 190 \mathrm{GeV}$.

Finally, we mention testability of our model at collider experiments and future directions of this scenario. Since the $\mathrm{DM}$ should be as heavy as a $\mathrm{TeV}$ range in order to satisfy the relic abundance and the constraints from the direct searches, the detection of the extra Higgs can be an important probe of our model similarly to the Higgs singlet model. On the benchmark points shown in Fig. 1, the mass of the additional Higgs boson is predicted to be in the range of $70-200 \mathrm{GeV}$, so that it can be produced at future lepton colliders such as the International Linear Collider (ILC) via the $\mathrm{Z}$ boson strahlung process. Therefore, our model can be tested at the ILC and/or its energy upgraded version. 

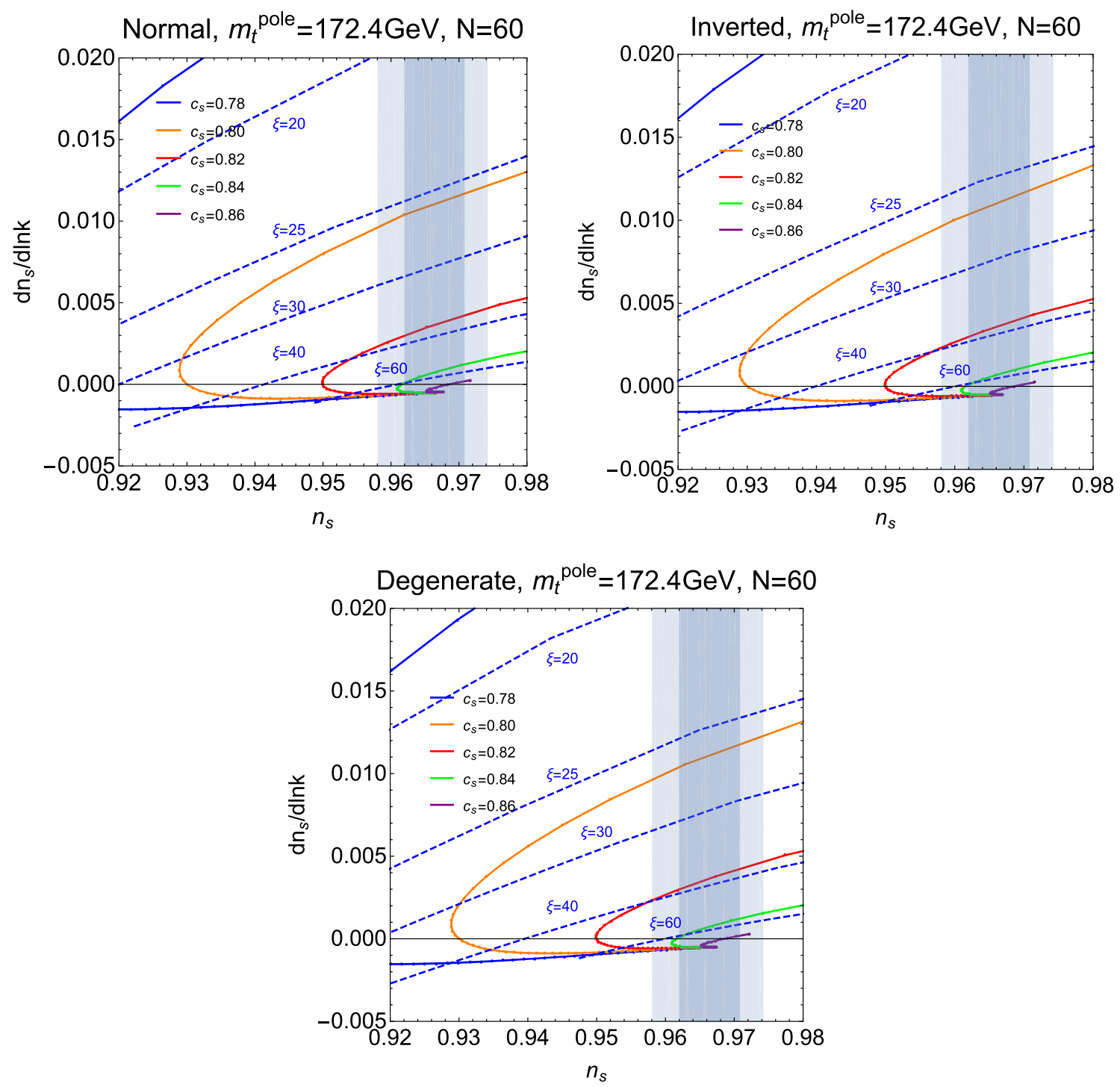

Fig. 7 Upper left (right): $n_{s}$ vs $d n_{s} / d \ln k$ in the case of the normal (inverted) hierarchy where $c(\xi)$ is fixed on each solid (dashed) curves. The blue regions correspond to the allowed regions by Planck 2018. Lower: the degenerate case

Our model can be well tested at the near future DM detection experiments such as XENONnT. If the whole region is excluded by them, one of the simplest extensions would be introduction of extra heavy fermions that are singlet under the SM gauge symmetry but are odd under $Z_{2}$ such that they lower the quartic scalar couplings in the RGE running to make the perturbativity bound milder.

It would be interesting to analyze all the possible criticalities along the line of the current work. Moreover, we can also come up with a lot of interesting phenomena within this model such as a possibility of producing primordial Black Hole by Higgs inflation [72-75], spontaneous leptogenesis [76-80], (p)reheating dynamics and so on. We would like to discuss those possibilities in future investigations.
Finally, we comment on possible systematic errors introduced by higher-order corrections. We have used the oneloop effective potential to discuss the MPP at the TeVscale, and two-loop RGEs to extrapolate from there to high scales. The higher-order corrections are further suppressed at approximately $\lambda_{S H} / 8 \pi^{2} \lesssim$ few percent, compared to the corrections currently considered. The same degree of corrections applies to the potential value $\propto \lambda_{H}$ and the inflationary predictions derived from it.

Acknowledgements The work of YH is supported by JSPS Overseas Research Fellowships. The work of KO is partly supported by the JSPS Kakenhi Grant No. 19H01899. The work of KY is supported in part by the Grant-in-Aid for Early-Career Scientists, No. 19K14714. 
Data Availability Statement This manuscript has no associated data or the data will not be deposited. [Authors' comment: This work is purely theoretical, and there is no background data we have used.]

Open Access This article is licensed under a Creative Commons Attribution 4.0 International License, which permits use, sharing, adaptation, distribution and reproduction in any medium or format, as long as you give appropriate credit to the original author(s) and the source, provide a link to the Creative Commons licence, and indicate if changes were made. The images or other third party material in this article are included in the article's Creative Commons licence, unless indicated otherwise in a credit line to the material. If material is not included in the article's Creative Commons licence and your intended use is not permitted by statutory regulation or exceeds the permitted use, you will need to obtain permission directly from the copyright holder. To view a copy of this licence, visit http://creativecomm ons.org/licenses/by/4.0/.

Funded by SCOAP ${ }^{3}$.

\section{Appendix A Two-Loop RGEs}

Here, we summarize the two-loop RGEs. Our calculations are based on [81-84].

$$
\begin{aligned}
\frac{d g_{Y}}{d t}= & \frac{1}{(4 \pi)^{2}} \frac{41}{6} g_{Y}^{3}+\frac{g_{Y}^{3}}{(4 \pi)^{4}} \frac{19}{6} \\
& \times\left(\frac{199}{18} g_{Y}^{2}+\frac{9}{2} g_{2}^{2}+\frac{44}{3} g_{3}^{2}-\frac{17}{6} y_{t}^{2}-\frac{n_{v}}{2} y_{v}^{2}\right) \\
\frac{d g_{2}}{d t}= & \frac{1}{(4 \pi)^{2}}\left(-\frac{19}{6}\right) g_{2}^{3}+\frac{g_{2}^{3}}{(4 \pi)^{4}} \frac{19}{6} \\
& \times\left(\frac{3}{2} g_{Y}^{2}+\frac{35}{6} g_{2}^{2}+12 g_{3}^{2}-\frac{3}{2} y_{t}^{2}-\frac{n_{v}}{2} y_{v}^{2}\right) \\
\frac{d g_{3}}{d t}= & \frac{1}{(4 \pi)^{2}}(-7) g_{2}^{3}+\frac{g_{2}^{3}}{(4 \pi)^{4}} \frac{19}{6} \\
& \times\left(\frac{11}{6} g_{Y}^{2}+\frac{9}{2} g_{2}^{2}-26 g_{3}^{2}-2 y_{t}^{2}\right) \\
\frac{d \lambda_{H}}{d t}= & \frac{1}{16 \pi^{2}}\left(24 \lambda_{H}^{2}+\frac{\lambda_{S H}^{2}}{2}+\frac{\lambda_{\phi H}^{2}}{2}-3 \lambda_{H}\left(g_{Y}^{2}+3 g_{2}^{2}\right)\right. \\
& +\frac{3}{8} g_{Y}^{4}+\frac{3}{4} g_{Y}^{2} g_{2}^{2}+\frac{9}{8} g_{2}^{4}+4 \lambda_{H}\left(3 y_{t}^{2}+n_{\nu} y_{v}^{2}\right) \\
& \left.-6 y_{t}^{4}-2 n_{\nu} y_{v}^{4}\right)+\frac{1}{(4 \pi)^{4}}\left\{-312 \lambda_{H}^{3}-2\right. \\
& \times \lambda_{\phi H}^{3}-5 \lambda_{H} \lambda_{\phi H}^{2}-2 \lambda_{S H}^{3}-5 \lambda_{H} \lambda_{S H}^{2}+36 \lambda_{H}^{2} \\
& \times\left(g_{Y}^{2}+3 g_{2}^{2}\right)-\lambda_{H}\left(-\frac{39}{4} g_{2}^{2} g_{Y}^{2}-\frac{629 g_{Y}^{4}}{24}\right. \\
& \left.+\frac{73 g_{2}^{4}}{8}\right)+\frac{305 g_{2}^{6}}{16}-\frac{289}{48} g_{2}^{4} g_{Y}^{2}-\frac{559}{48} g_{2}^{2} g_{Y}^{4} \\
& -\frac{379 g_{Y}^{6}}{48}-32 g_{3}^{2} y_{t}^{4}-\frac{8}{3} g_{Y}^{2} y_{t}^{4}-\frac{9}{4} g_{2}^{4}\left(y_{t}^{2}+y_{v}^{2}\right)
\end{aligned}
$$

$$
\begin{aligned}
& \times\left(3 y_{t}^{2}+n_{v} y_{v}^{2}\right)-\frac{9}{2}\left(3 y_{t}^{4}+n_{v} y_{v}^{4}\right)+g_{Y}^{2} \\
& \times\left(\lambda_{\phi H}+24 \lambda_{H}\right)+3 g_{2}^{2}\left(\lambda_{\phi H}+24 \lambda_{H}\right) \\
& +y_{t}^{2}\left(\frac{85 g_{Y}^{2}}{12}+\frac{45 g_{2}^{2}}{4}+40 g_{3}^{2}\right)+n_{v} y_{v}^{2} \\
& \left.\times\left(\frac{5 g_{Y}^{2}}{4}+\frac{15 g_{2}^{2}}{4}\right)-\frac{145}{16} g_{2}^{4}+\frac{15}{8} g_{2}^{2} g_{Y}^{2}+\frac{557}{48} g_{Y}^{4}\right\} \\
& +\frac{1}{(4 \pi)^{4}}\left(-2 \lambda_{\phi S} \lambda_{S H}^{2}-2 \lambda_{\phi S}^{2} \lambda_{S H}\right), \\
\frac{d \lambda_{S H}}{d t}= & \frac{\lambda_{S H}}{16 \pi^{2}}\left(12 \lambda_{H}+\lambda_{S}+4 \lambda_{S H}+6 y_{t}^{2}+2 n_{v} y_{v}^{2}\right. \\
& \left.-\frac{3}{2} g_{Y}^{2}-\frac{9}{2} g_{2}^{2}\right)-\frac{\lambda_{\phi H} \lambda_{\phi S}}{16 \pi^{2}}
\end{aligned}
$$




$$
\begin{aligned}
& +\frac{\lambda_{S H}}{(4 \pi)^{4}}\left\{-\frac{21 \lambda_{S H}^{2}}{2}-72 \lambda_{H} \lambda_{S H}\right. \\
& -60 \lambda_{H}^{2}-6 \lambda_{S} \lambda_{S H}-\frac{5}{6} \lambda_{S}^{2}-\frac{1}{2} \lambda_{\phi S}^{2} \\
& -4 \lambda_{\phi S} \lambda_{\phi H}-\frac{1}{2} \lambda_{\phi H}^{2} \\
& -\left(4 \lambda_{S H}+24 \lambda_{H}\right)\left(3 y_{t}^{2}+n_{v} y_{v}^{2}\right) \\
& -\frac{9}{2}\left(3 y_{t}^{4}+n_{v} y_{v}^{4}\right)+g_{Y}^{2}\left(\lambda_{S H}+24 \lambda_{H}\right) \\
& +3 g_{2}^{2}\left(\lambda_{S H}+24 \lambda_{H}\right) \\
& +y_{t}^{2}\left(\frac{85 g_{Y}^{2}}{12}+\frac{45 g_{2}^{2}}{4}+40 g_{3}^{2}\right) \\
& +n_{v} y_{v}^{2}\left(\frac{5 g_{Y}^{2}}{4}+\frac{15 g_{2}^{2}}{4}\right)-\frac{145}{16} g_{2}^{4}+\frac{15}{8} g_{2}^{2} g_{Y}^{2} \\
& \left.+\frac{557}{48} g_{Y}^{4}\right\}+\frac{1}{(4 \pi)^{4}}\left(-2 \lambda_{\phi H}^{2} \lambda_{\phi S}-2 \lambda_{\phi H} \lambda_{\phi S}^{2}\right),
\end{aligned}
$$$$
\frac{d \lambda_{\phi S}}{d t}=\frac{\lambda_{\phi S}}{16 \pi^{2}}\left(\lambda_{\phi}+\lambda_{S}+4 \lambda_{\phi S}\right)
$$$$
-\frac{\lambda_{\phi H} \lambda_{S H}}{16 \pi^{2}}+\frac{\lambda_{\phi S}}{(4 \pi)^{4}}\left\{-9 \lambda_{\phi S}^{2}\right.
$$$$
-6 \lambda_{\phi S}\left(\lambda_{\phi}+\lambda_{S}\right)-\frac{5}{6}\left(\lambda_{\phi}^{2}+\lambda_{S}^{2}\right)
$$$$
\left.-2\left(\lambda_{\phi H}^{2}+\lambda_{S H}^{2}\right)-16 \lambda_{\phi H} \lambda_{S H}\right\}
$$$$
+\frac{\lambda_{\phi H} \lambda_{S H}}{(4 \pi)^{4}}\left\{-8\left(3 y_{t}^{2}+n_{\nu} y_{v}^{2}\right)\right.
$$$$
\left.\times+8\left(g_{Y}^{2}+3 g_{2}^{2}\right)-8\left(\lambda_{S H}+\lambda_{\phi H}\right)\right\},
$$$$
\frac{d y_{t}}{d t}=\frac{y_{t}}{16 \pi^{2}}\left(\frac{9}{2} y_{t}^{2}+n_{v} y_{v}^{2}-\frac{17}{12} g_{y}^{2}-\frac{9}{4} g_{2}^{2}-8 g_{3}^{2}\right)
$$$$
+\frac{y_{t}}{(4 \pi)^{4}}\left\{-12 y_{t}^{4}-\frac{9 n_{v}}{4} y_{t}^{2} y_{v}^{2}-\frac{9 n_{v}}{4} y_{v}^{4}\right.
$$$$
+6 \lambda_{H}^{2}+\frac{\lambda_{\phi H}^{2}}{4}+\frac{\lambda_{S H}^{2}}{4}
$$$$
-12 \lambda_{H} y_{t}^{2}+\frac{131}{16} g_{Y}^{2} y_{t}^{2}+\frac{225}{16} g_{2}^{2} y_{t}^{2}+36 g_{3}^{2} y_{t}^{2}
$$$$
+n_{v}\left(\frac{15}{8} g_{2}^{2}+\frac{5}{8} g_{Y}^{2}\right) y_{v}^{2}
$$$$
+\frac{1187 g_{Y}^{4}}{216}-\frac{23 g_{2}^{4}}{4}-108 g_{3}^{4}
$$$$
\left.-\frac{3}{4} g_{2}^{2} g_{Y}^{2}+9 g_{3}^{2} g_{2}^{2}+\frac{19}{9} g_{3}^{2} g_{Y}^{2}\right\},
$$$$
\frac{d y_{v}}{d t}=\frac{y_{v}}{16 \pi^{2}}\left(\left(n_{v}+\frac{3}{2}\right) y_{v}^{2}+3 y_{t}^{2}-\frac{3}{4} g_{y}^{2}-\frac{9}{4} g_{2}^{2}\right)
$$$$
+\frac{y_{v}}{(4 \pi)^{4}}\left\{-\left(\frac{9 n_{v}}{2}-\frac{3}{2}\right) y_{v}^{4}-\frac{27}{4} y_{t}^{2} y_{v}^{2}-\frac{27}{4} y_{t}^{4}\right.
$$

$$
\frac{d n_{s}}{d \ln k}=0.013 \pm 0.024, \quad \quad(68 \% \mathrm{CL})
$$

at the pivot scale $k_{*}=0.05 \mathrm{Mpc}^{-1}$.

Under the slow-roll approximation, the scalar amplitude $A_{s}$ is given by Eq. (67). We note that $r$ and the value of 
potential $U$ is related each other by fixing $A_{s}$ to the observed value:

$U \sim 1.5 \times 10^{-9}\left[\frac{r}{0.05}\right] M_{\mathrm{P}}^{4}$

see also Ref. [22].

\section{Appendix C Ordinary Higgs inflation without criticality}

For $\frac{\xi h^{2}}{M_{\mathrm{P}}^{2}} \gg 1$ we have simple relations for the slow roll parameters

$\varepsilon_{V} \simeq \frac{4}{3} \exp \left(-2 \sqrt{\frac{2}{3}} \frac{\chi}{M_{\mathrm{P}}}\right) \simeq \frac{3}{4 N^{2}}$,

$\eta_{V} \simeq-\frac{4}{3} \exp \left(-\sqrt{\frac{2}{3}} \frac{\chi}{M_{\mathrm{P}}}\right)=-\frac{1}{N}$,

and they provide one of the best fits to the CMB observations for the reasonable values of e-folding $N=50-60$ (corresponding to the pivot scale).

Qualitatively, the typical value of $\xi$ can be estimated as follows. Putting the potential (51) with $\lambda_{\text {eff }} \sim \lambda_{c} \sim 10^{-6}$ into Eq. (69), one can easily check that $\xi$ is around 30.

\section{Appendix D Expansion around saddle point}

For qualitative understanding, it is also helpful to derive the expansion of $V$ around $h_{s}$. We first expand as

$$
\begin{aligned}
V= & \frac{\lambda_{c}}{2} h_{s}^{4}+\lambda_{1} h_{s}^{3}\left(h-h_{s}\right)+\frac{\lambda_{2}}{2} h_{s}^{2}\left(h-h_{s}\right)^{2} \\
& +\frac{\lambda_{3}}{3 !} h_{s}\left(h-h_{s}\right)^{3}+\ldots,
\end{aligned}
$$

where

$\lambda_{1}=\lambda_{c} \delta, \quad \lambda_{2}=3 \lambda_{c} \delta, \quad \lambda_{3}=32 \lambda_{c}$.

As we explain in Sect. 4.2, the Higgs potential in the Einstein frame also has a saddle point at $h=h_{s} \Omega$. We are interested in the parameter space $c_{s} \sim 1 \Leftrightarrow \tilde{h}_{s} \gg h_{s} \sim \frac{M_{\mathrm{P}}}{\sqrt{\xi}}$, which guarantees the large field expansion of $\chi$ as a function of $h$. As a result, we have

$$
\begin{aligned}
& \frac{d h}{d \chi} \sim \frac{h}{\sqrt{6} M_{\mathrm{P}}} \Leftrightarrow h \sim \frac{M_{\mathrm{P}}}{\sqrt{\xi}} \exp \left(\frac{\chi}{\sqrt{6} M_{\mathrm{P}}}\right), \\
& \varphi \sim \frac{M_{\mathrm{P}}}{\sqrt{\xi}}\left(1-\frac{\left(\frac{M_{\mathrm{P}}}{\sqrt{\xi}}\right)^{2}}{2 h^{2}}\right) \Leftrightarrow \varphi-h_{s} \sim \frac{M_{\mathrm{P}}}{\sqrt{\xi}}
\end{aligned}
$$

$$
\times\left[1-c-\frac{1}{2} e^{-\frac{2 \chi}{\sqrt{6} M_{\mathrm{P}}}}\right]
$$

where $\varphi:=h / \Omega$. Then, $U$ and its derivatives with respect to $\chi$ are

$$
\begin{aligned}
U \sim & \frac{\lambda_{c}}{4} h_{s}^{4}=\frac{\lambda_{0} c^{4}}{4}\left(\frac{M_{\mathrm{P}}}{\sqrt{\xi}}\right)^{4} \\
& \frac{\partial U}{\partial \chi}=\frac{\partial \varphi}{\partial \chi} \frac{\partial U}{\partial \varphi} \\
& \sim \frac{\frac{M_{\mathrm{P}}}{\sqrt{\xi}} e^{-2 \chi /\left(\sqrt{6} M_{\mathrm{P}}\right)}}{\sqrt{6} M_{\mathrm{P}}}\left(\lambda_{1} h_{s}^{3}+\lambda_{2} h_{s}^{2}\left(\varphi-h_{s}\right)\right. \\
& \left.+\frac{\lambda_{3} h_{s}}{2}\left(\varphi-h_{s}\right)^{2}\right) \\
& \sim \frac{\lambda_{3} \frac{M_{\mathrm{P}}}{\sqrt{\xi}} h_{s}^{3}}{\sqrt{6} M_{\mathrm{P}}} e^{-2 \chi /\left(\sqrt{6} M_{\mathrm{P}}\right)}\left[\frac{\delta}{32}+\frac{3 \delta}{32 c}\right. \\
& \left.\times\left(1-c-\frac{1}{2} e^{-\frac{2 \chi}{\sqrt{6} M_{\mathrm{P}}}}\right)+\frac{1}{2 c^{2}}\left(1-c-\frac{1}{2} e^{-\frac{2 \chi}{\sqrt{6} M_{\mathrm{P}}}}\right)^{2}\right],
\end{aligned}
$$

$$
\begin{aligned}
& \frac{\partial^{2} U}{\partial \chi^{2}} \sim-\frac{\lambda_{3} \frac{M_{\mathrm{P}}}{\sqrt{\xi}} h_{s}^{3}}{3 M_{\mathrm{P}}^{2}} e^{-2 \chi /\left(\sqrt{6} M_{\mathrm{P}}\right)}\left[\frac{\delta}{32}\right. \\
& +\frac{3 \delta}{32 c}\left(1-c-\frac{1}{2} e^{-\frac{2 \chi}{\sqrt{6} M_{\mathrm{P}}}}\right)+\frac{1}{2 c^{2}} \\
& \times\left(1-c-\frac{1}{2} e^{-\frac{2 \chi}{\sqrt{6} M_{\mathrm{P}}}}\right)^{2}-\frac{e^{-2 \chi /\left(\sqrt{6} M_{\mathrm{P}}\right)}}{2} \\
& \left.\left\{\frac{3 \delta}{32}+\frac{1}{c^{2}}\left(1-c-\frac{1}{2} e^{-\frac{2 \chi}{\sqrt{6} M_{\mathrm{P}}}}\right)\right\}\right]
\end{aligned}
$$

where we have used Eq. (71). When $c \sim 1$, the slow roll parameters are approximately given by

$\varepsilon=\frac{M_{\mathrm{P}}^{2}}{2}\left(\frac{U^{\prime}}{U}\right)^{2} \sim \frac{4}{3}\left(\frac{\delta}{c}\right)^{2} e^{-4 \chi /\left(\sqrt{6} M_{\mathrm{P}}\right)}$,

$\eta=M_{\mathrm{P}}^{2} \frac{U^{\prime \prime}}{U} \sim-\frac{4 \delta}{3 c} e^{-2 \chi /\left(\sqrt{6} M_{\mathrm{P}}\right)}$,

where $a=\lambda_{1} / \lambda_{0}=1+\left.\left(\beta_{\lambda} / 4 \lambda\right)\right|_{\phi=\phi_{s}}$. Compared to the conventional case, we have additional suppression factor $\delta$ thanks to the saddle potential. Note that, when $c \ll 1$, we can no longer trust Eq. (78).

\section{References}

1. Y. Hamada, H. Kawai, K.-Y. Oda, S.C. Park, Higgs inflation from Standard Model criticality. Phys. Rev. D 91, 053008 (2015). arXiv:1408.4864 [hep-ph]

2. G. Degrassi, S. Di Vita, J. Elias-Miro, J.R. Espinosa, G.F. Giudice, G. Isidori, A. Strumia, Higgs mass and vacuum stability in the 
Standard Model at NNLO. JHEP 08, 098 (2012). arXiv:1205.6497 [hep-ph]

3. D. Buttazzo, G. Degrassi, P.P. Giardino, G.F. Giudice, F. Sala, A. Salvio, A. Strumia, Investigating the near-criticality of the Higgs boson. JHEP 12, 089 (2013). arXiv:1307.3536 [hep-ph]

4. A. Bednyakov, B. Kniehl, A. Pikelner, O. Veretin, Stability of the electroweak vacuum: gauge independence and advanced precision. Phys. Rev. Lett. 115(20), 201802 (2015). arXiv:1507.08833 [hep$\mathrm{ph}]$

5. P.Z. et al. (Particle Data Group), The review of particle physics. Prog. Theor. Exp. Phys. 2020, 083 C01 (2020)

6. C. Froggatt, H.B. Nielsen, Standard model criticality prediction: Top mass $173+-5-\mathrm{GeV}$ and Higgs mass $135+-9-\mathrm{GeV}$. Phys. Lett. B 368, 96-102 (1996). arXiv:hep-ph/9511371

7. C. Froggatt, H.B. Nielsen, Y. Takanishi, Standard model Higgs boson mass from borderline metastability of the vacuum. Phys. Rev. D 64, 113014 (2001). arXiv:hep-ph/0104161

8. H.B. Nielsen, PREdicted the Higgs mass. Bled Workshops Phys. 13(2), 94-126 (2012). arXiv:1212.5716 [hep-ph]

9. H. Kawai, T. Okada, Asymptotically vanishing cosmological constant in the multiverse. Int. J. Mod. Phys. A 26, 3107-3120 (2011). arXiv:1104.1764 [hep-th]

10. H. Kawai, T. Okada, Solving the naturalness problem by baby universes in the Lorentzian multiverse. Prog. Theor. Phys. 127, 689721 (2012). arXiv:1110.2303 [hep-th]

11. H. Kawai, Low energy effective action of quantum gravity and the naturalness problem. Int. J. Mod. Phys. A 28, 1340001 (2013)

12. Y. Hamada, H. Kawai, K. Kawana, Evidence of the Big Fix. Int. J. Mod. Phys. A 29, 1450099 (2014). arXiv:1405.1310 [hep-ph]

13. Y. Hamada, H. Kawai, K. Kawana, Weak scale from the maximum entropy principle. PTEP 2015, 033B06 (2015). arXiv:1409.6508 [hep-ph]

14. Y. Hamada, H. Kawai, K. Kawana, Natural solution to the naturalness problem: the universe does fine-tuning. PTEP 2015(12), 123B03 (2015). arXiv:1509.05955 [hep-th]

15. Y. Hamada, H. Kawai, K.-Y. Oda, Eternal Higgs inflation and the cosmological constant problem. Phys. Rev. D 92, 045009 (2015). arXiv:1501.04455 [hep-ph]

16. K. Kannike, N. Koivunen, M. Raidal, Principle of multiple point criticality in multi-scalar dark matter models. arXiv:2010.09718 [hep-ph]

17. D. Bennett, H.B. Nielsen, I. Picek, Understanding fine structure constants and three generations. Phys. Lett. B 208, 275-280 (1988)

18. D. Bennett, H.B. Nielsen, Predictions for nonAbelian fine structure constants from multicriticality. Int. J. Mod. Phys. A 9, 5155-5200 (1994). arXiv:hep-ph/9311321

19. Y. Hamada, H. Kawai, K.-Y. Oda, S.C. Park, Higgs inflation is still alive after the results from BICEP2. Phys. Rev. Lett. 112(24), 241301 (2014). arXiv:1403.5043 [hep-ph]

20. F. Bezrukov, M. Shaposhnikov, Higgs inflation at the critical point. Phys. Lett. B 734, 249-254 (2014). arXiv:1403.6078 [hep-ph]

21. Y. Hamada, H. Kawai, K.-Y. Oda, Predictions on mass of Higgs portal scalar dark matter from Higgs inflation and flat potential. JHEP 07, 026 (2014). arXiv: 1404.6141 [hep-ph]

22. Y. Hamada, H. Kawai, Y. Nakanishi, K.-Y. Oda, Cosmological implications of Standard Model criticality and Higgs inflation. Nucl. Phys. B 953, 114946 (2020). arXiv:1709.09350 [hep-ph]

23. P. Minkowski, $\mu \rightarrow e \gamma$ at a rate of one out of $10^{9}$ muon decays? Phys. Lett. B 67, 421-428 (1977)

24. T. Yanagida, Horizontal gauge symmetry and masses of neutrinos. Conf. Proc. C 7902131, 95-99 (1979)

25. M. Gell-Mann, P. Ramond, R. Slansky, Complex spinors and unified theories. Conf. Proc. C 790927, 315-321 (1979). arXiv: 1306.4669 [hep-th]

26. S. Glashow, The future of elementary particle physics. NATO Sci. Ser. B 61, 687 (1980)
27. R.N. Mohapatra, G. Senjanovic, Neutrino mass and spontaneous parity nonconservation. Phys. Rev. Lett. 44, 912 (1980)

28. F.L. Bezrukov, M. Shaposhnikov, The Standard Model Higgs boson as the inflation. Phys. Lett. B 659, 703-706 (2008). arXiv:0710.3755 [hep-th]

29. A. Barvinsky, A. Kamenshchik, A. Starobinsky, Inflation scenario via the Standard Model Higgs boson and LHC. JCAP 11, 021 (2008). arXiv:0809.2104 [hep-ph]

30. A. De Simone, M.P. Hertzberg, F. Wilczek, Running inflation in the Standard Model. Phys. Lett. B 678, 1-8 (2009). arXiv:0812.4946 [hep-ph]

31. K. Allison, Higgs xi-inflation for the $125-126 \mathrm{GeV}$ Higgs: a twoloop analysis. JHEP 02, 040 (2014). arXiv:1306.6931 [hep-ph]

32. C. Burgess, H.M. Lee, M. Trott, Power-counting and the validity of the classical approximation during inflation. JHEP 09, 103 (2009). arXiv:0902.4465 [hep-ph]

33. J. Barbon, J. Espinosa, On the naturalness of Higgs inflation. Phys. Rev. D 79, 081302 (2009). arXiv:0903.0355 [hep-ph]

34. C. Burgess, H.M. Lee, M. Trott, Comment on Higgs inflation and naturalness. JHEP 07, 007 (2010). arXiv:1002.2730 [hep-ph]

35. F. Bezrukov, A. Magnin, M. Shaposhnikov, S. Sibiryakov, Higgs inflation: consistency and generalisations. JHEP 01, 016 (2011). arXiv: 1008.5157 [hep-ph]

36. Y. Ema, R. Jinno, K. Mukaida, K. Nakayama, Violent preheating in inflation with nonminimal coupling. JCAP 02, 045 (2017). arXiv:1609.05209 [hep-ph]

37. J. Kubo, J. Kuntz, M. Lindner, J. Rezacek, P. Saake, A. Trautner, Unified emergence of energy scales and cosmic inflation. arXiv:2012.09706 [hep-ph]

38. V. Silveira, A. Zee, Scalar phantoms. Phys. Lett. B 161, 136-140 (1985)

39. J. McDonald, Gauge singlet scalars as cold dark matter. Phys. Rev. D 50, 3637-3649 (1994). arXiv:hep-ph/0702143

40. C. Burgess, M. Pospelov, T. ter Veldhuis, The minimal model of nonbaryonic dark matter: a singlet scalar. Nucl. Phys. B 619, 709_ 728 (2001). arXiv:hep-ph/0011335

41. J.M. Cline, K. Kainulainen, P. Scott, C. Weniger, Update on scalar singlet dark matter. Phys. Rev. D 88, 055025 (2013). arXiv:1306.4710 [hep-ph] (Erratum: Phys. Rev. D 92, 039906 (2015))

42. Y. Hamada, H. Kawai, K.-Y. Oda, Minimal Higgs inflation. PTEP 2014, 023B02 (2014). arXiv:1308.6651 [hep-ph]

43. S.R. Coleman, E.J. Weinberg, Radiative corrections as the origin of spontaneous symmetry breaking. Phys. Rev. D 7, 1888-1910 (1973)

44. K.A. Meissner, H. Nicolai, Conformal symmetry and the Standard Model. Phys. Lett. B 648, 312-317 (2007). arXiv:hep-th/0612165

45. R. Foot, A. Kobakhidze, K.L. McDonald, R.R. Volkas, A solution to the hierarchy problem from an almost decoupled hidden sector within a classically scale invariant theory. Phys. Rev. D 77, 035006 (2008). arXiv:0709.2750 [hep-ph]

46. S. Iso, N. Okada, Y. Orikasa, Classically conformal $B-L$ extended Standard Model. Phys. Lett. B 676, 81-87 (2009). arXiv:0902.4050 [hep-ph]

47. S. Iso, N. Okada, Y. Orikasa, The minimal $B-L$ model naturally realized at TeV scale. Phys. Rev. D 80, 115007 (2009). arXiv:0909.0128 [hep-ph]

48. T. Hur, P. Ko, Scale invariant extension of the standard model with strongly interacting hidden sector. Phys. Rev. Lett. 106, 141802 (2011). arXiv:1103.2571 [hep-ph]

49. S. Iso, Y. Orikasa, TeV Scale B-L model with a flat Higgs potential at the Planck scale: in view of the hierarchy problem. PTEP 2013, 023B08 (2013). arXiv:1210.2848 [hep-ph]

50. C. Englert, J. Jaeckel, V. Khoze, M. Spannowsky, Emergence of the electroweak scale through the Higgs portal. JHEP 04, 060 (2013). arXiv:1301.4224 [hep-ph] 
51. M. Hashimoto, S. Iso, Y. Orikasa, Radiative symmetry breaking at the Fermi scale and flat potential at the Planck scale. Phys. Rev. D 89, 016019 (2014). arXiv:1310.4304 [hep-ph]

52. M. Holthausen, J. Kubo, K.S. Lim, M. Lindner, Electroweak and conformal symmetry breaking by a strongly coupled hidden sector. JHEP 12, 076 (2013). arXiv:1310.4423 [hep-ph]

53. M. Hashimoto, S. Iso, Y. Orikasa, Radiative symmetry breaking from flat potential in various U(1)' models. arXiv:1401.5944 [hep$\mathrm{ph}]$

54. J. Kubo, K.S. Lim, M. Lindner, Electroweak symmetry breaking via QCD. Phys. Rev. Lett. 113, 091604 (2014). arXiv:1403.4262 [hep-ph]

55. J. Kubo, M. Yamada, Genesis of electroweak and dark matter scales from a bilinear scalar condensate. Phys. Rev. D 93(7), 075016 (2016). arXiv:1505.05971 [hep-ph]

56. D.-W. Jung, J. Lee, S.-H. Nam, Scalar dark matter in the conformally invariant extension of the standard model. Phys. Lett. B 797, 134823 (2019). arXiv:1904.10209 [hep-ph]

57. P.H. Chankowski, A. Lewandowski, K.A. Meissner, H. Nicolai, Softly broken conformal symmetry and the stability of the electroweak scale. Mod. Phys. Lett. A 30(02), 1550006 (2015). arXiv: 1404.0548 [hep-ph]

58. K.A. Meissner, H. Nicolai, J. Plefka, Softly broken conformal symmetry with quantum gravitational corrections. Phys. Lett. B 791, 62-65 (2019). arXiv:1811.05216 [hep-th]

59. M. Veltman, The infrared-ultraviolet connection. Acta Phys. Pol. B 12, 437 (1981)

60. Y. Hamada, H. Kawai, K.-Y. Oda, Bare Higgs mass at Planck scale. Phys. Rev. D 87(5), 053009 (2013). arXiv:1210.2538 [hep-ph] (Erratum: Phys.Rev.D 89, 059901 (2014))

61. J. Haruna, H. Kawai, Weak scale from Planck scale: mass scale generation in a classically conformal two-scalar system. PTEP 2020(3), 033B01 (2020). arXiv:1905.05656 [hep-th]

62. Y. Hamada, H. Kawai, K.-Y. Oda, K. Yagyu, Dark matter in minimal dimensional transmutation with multipoint criticality principle. arXiv:2008.08700 [hep-ph]

63. Planck Collaboration, N. Aghanim et al., Planck 2018 results. VI. Cosmological parameters. Astron. Astrophys. 641, A6 (2020). arXiv:1807.06209 [astro-ph.CO]

64. XENON Collaboration, E. Aprile et al., Dark matter search results from a one ton-year exposure of XENON1T. Phys. Rev. Lett. 121(11), 111302 (2018). arXiv:1805.12562 [astro-ph.CO]

65. C.P. Burgess, Introduction to effective field theory. Ann. Rev. Nucl. Part. Sci. 57, 329-362 (2007). arXiv:hep-th/0701053

66. S. Iso, K. Kawana, RG-improvement of the effective action with multiple mass scales. JHEP 03, 165 (2018). arXiv:1801.01731 [hep-ph]

67. M. Bando, T. Kugo, N. Maekawa, H. Nakano, Improving the effective potential: multimass scale case. Prog. Theor. Phys. 90, 405-418 (1993). arXiv:hep-ph/9210229
68. G. Isidori, V.S. Rychkov, A. Strumia, N. Tetradis, Gravitational corrections to standard model vacuum decay. Phys. Rev. D 77, 025034 (2008). arXiv:0712.0242 [hep-ph]

69. M. Fairbairn, P. Grothaus, R. Hogan, The problem with false vacuum Higgs inflation. JCAP 06, 039 (2014). arXiv:1403.7483 [hep$\mathrm{ph}]$

70. D. Salopek, J. Bond, J.M. Bardeen, Designing density fluctuation spectra in inflation. Phys. Rev. D 40, 1753 (1989)

71. Y. Hamada, H. Kawai, Y. Nakanishi, K.-Y. Oda, Meaning of the field dependence of the renormalization scale in Higgs inflation. Phys. Rev. D 95(10), 103524 (2017). arXiv:1610.05885 [hep-th]

72. J.M. Ezquiaga, J. Garcia-Bellido, E.R. Morales, Primordial black hole production in critical Higgs inflation. Phys. Lett. B 776, 345349 (2018). arXiv:1705.04861 [astro-ph.CO]

73. F. Bezrukov, M. Pauly, J. Rubio, On the robustness of the primordial power spectrum in renormalized Higgs inflation. JCAP 02, 040 (2018). arXiv:1706.05007 [hep-ph]

74. S. Rasanen, E. Tomberg, Planck scale black hole dark matter from Higgs inflation. JCAP 01, 038 (2019). arXiv:1810.12608 [astroph.CO]

75. D.Y. Cheong, S.M. Lee, S.C. Park, Primordial black holes in Higgs$R^{2}$ inflation as a whole dark matter. arXiv:1912.12032 [hep-ph]

76. S.M. Lee, K.-Y. Oda, S.C. Park, Spontaneous leptogenesis in Higgs inflation. arXiv:2010.07563 [hep-ph]

77. A. Kusenko, L. Pearce, L. Yang, Postinflationary Higgs relaxation and the origin of matter-antimatter asymmetry. Phys. Rev. Lett. 114(6), 061302 (2015). arXiv:1410.0722 [hep-ph]

78. A.G. Cohen, D.B. Kaplan, Thermodynamic generation of the Baryon asymmetry. Phys. Lett. B 199, 251-258 (1987)

79. A.G. Cohen, D.B. Kaplan, Spontaneous baryogenesis. Nucl. Phys. B 308, 913-928 (1988)

80. A. Dolgov, K. Freese, Calculation of particle production by Nambu goldstone bosons with application to inflation reheating and baryogenesis. Phys. Rev. D 51, 2693-2702 (1995). arXiv:hep-ph/9410346

81. M.E. Machacek, M.T. Vaughn, Two loop renormalization group equations in a general quantum field theory. 1 . Wave function renormalization. Nucl. Phys. B 222, 83-103 (1983)

82. M.E. Machacek, M.T. Vaughn, Two loop renormalization group equations in a general quantum field theory. 2. Yukawa couplings. Nucl. Phys. B 236, 221-232 (1984)

83. M.E. Machacek, M.T. Vaughn, Two loop renormalization group equations in a general quantum field theory. 3. Scalar quartic couplings. Nucl. Phys. B 249, 70-92 (1985)

84. M.-X. Luo, H.-W. Wang, Y. Xiao, Two loop renormalization group equations in general gauge field theories. Phys. Rev. D 67, 065019 (2003). arXiv:hep-ph/0211440

85. Planck Collaboration, Y. Akrami et al., Planck 2018 results. X. Constraints on inflation. Astron. Astrophys. 641, A10 (2020). arXiv:1807.06211 [astro-ph.CO] 